\title{
Article \\ Efficient Synthesis of a New Family of 2,6-Disulfanyl-9-selenabicyclo[3.3.1]nonanes
}

\author{
Maxim V. Musalov (D), Vladimir A. Potapov*(i) and Svetlana V. Amosova \\ A. E. Favorsky Irkutsk Institute of Chemistry, Siberian Division of The Russian Academy of Sciences, \\ 1 Favorsky Str., 664033 Irkutsk, Russia; musalov_maxim@irioch.irk.ru (M.V.M.); amosova@irioch.irk.ru (S.V.A.) \\ * Correspondence: v.a.potapov@mail.ru
}

Citation: Musalov, M.V.; Potapov, V.A.; Amosova, S.V. Efficient Synthesis of a New Family of 2,6-Disulfanyl-9selenabicyclo[3.3.1]nonanes. Molecules 2021, 26, 2849. https:// doi.org/10.3390/molecules26102849

Academic Editors: Scott K. Bur and Qiu Wang

Received: 19 April 2021

Accepted: 6 May 2021

Published: 11 May 2021

Publisher's Note: MDPI stays neutral with regard to jurisdictional claims in published maps and institutional affiliations.

Copyright: (c) 2021 by the authors. Licensee MDPI, Basel, Switzerland. This article is an open access article distributed under the terms and conditions of the Creative Commons Attribution (CC BY) license (https:// creativecommons.org/licenses/by/ $4.0 /)$.
Abstract: The efficient synthesis of a new family of 2,6-disulfanyl-9-selenabicyclo[3.3.1]nonanes in high yields has been developed based on 9-selenabicyclo[3.3.1]nonane-2,6-dithiolate anion generated from bis-isothiouronium salt of 2,6-dibromo-9-selenabicyclo[3.3.1]nonane. The derivatives of 2,6-disulfanyl-9-selenabicyclo[3.3.1]nonane containing alkyl, allyl and benzyl moieties have been prepared in 90-99\% yields by nucleophilic substitution of 9-selenabicyclo[3.3.1]nonane-2,6dithiolate anion with alkyl, allyl and benzyl halides. The reaction of nucleophilic addition of 9-selenabicyclo[3.3.1]nonane-2,6-dithiolate anion to alkyl propiolates afforded 2,6-di(vinylsulfanyl)-9selenabicyclo[3.3.1]nonanes. The conditions for regio- and stereoselective addition of 9-selenabicyclo [3.3.1]nonane-2,6-dithiolate anion to a triple bond of alkyl propiolates have been found. To date, not a single representative of 2,6-disulfanyl-9-selenabicyclo[3.3.1]nonanes has been described in the literature.

Keywords: 2,6-disulfanyl-9-selenabicyclo[3.3.1]nonanes; transannular addition; 9-selenabicyclo [3.3.1]nonane-2,6-dithiolate anion; isothiouronium salts; 2,6-dibromo-9-selenabicyclo[3.3.1]nonane; selenium dibromide

\section{Introduction}

The importance of chemistry of heterocyclic compounds for the development of organic medicinal and pharmaceutical chemistry is difficult to overestimate. A lion's share of modern drugs contains heterocyclic moieties in their structures [1,2]. The discovery of many novel drugs is closely related to the development of chemistry of heterocyclic compounds. Heterocyclic derivatives exhibit various types of biological activity [1,2]. Many distinguished scientists have made important contributions to modern chemistry of heterocyclic compounds [1-4].

Selenium is a micronutrient for mammals and an essential trace element nutrient for humans that functions as cofactor for glutathione peroxidase and certain forms of thioredoxin reductase [5-7]. Organoselenium heterocycles display a variety of biological activities, including antibacterial, antifungal, antitumor, anti-inflammatory, neuroprotective and glutathione peroxidase-like actions [8-15].

Selenium heterocyclic compound Ebselen shows anti-inflammatory, neuroprotective and glutathione peroxidase-like activities [13-15]. This compound finds application as an anti-inflammatory agent. Ebselen is also used for the treatment and prevention of cardiovascular diseases and ischemic stroke.

The anchimeric assistance effect of selenium in comparison with the effect of sulfur and nitrogen atoms has been quantitatively estimated using 2,6-dichloro-9-selenabicyclo [3.3.1]nonane, 2,6-dichloro-9-thia- and 2,6-dichloro-9-azabicyclo[3.3.1]nonane as model substrates [16]. Based on the determination of the absolute and relative rates of nucleophilic substitution of chlorine in these compounds, it has been established that the anchimeric assistance effect of the selenium atom is more than two orders of magnitude greater than the effect of the sulfur and nitrogen atoms. 2,6-Dichloro-9-selenabicyclo[3.3.1]nonane 
has been obtained by the transannular addition of selenium dichloride to cis,cis-1,5cyclooctadiene [16].

The biochemical potential of 9-selenabicyclo[3.3.1]nonanes has not yet been revealed; however, it is known that its sulfur and nitrogen analogues exhibit a variety of biological activities [17-27].

Antimicrobial coatings containing the 9-thiabicyclo[3.3.1]nonane moiety have been developed [17]. The resulting surfaces displayed antibacterial and antifungal activities.

The 9-thiabicyclo[3.3.1]nonane derivatives, which were obtained by nucleophilic substitution of halogen in 2,6-dichloro-9-thiabicyclo[3.3.1]nonane, displayed anti-inflammatory activity [18] (Figure 1). Polycation polymers containing the 9-thiabicyclo[3.3.1]nonane unit showed antimicrobial activity [19] (Figure 1). These polymers inhibited the growth of bacteria at low concentration (e.g., the minimum inhibitory concentration in PBS buffer is $0.12-0.5 \mu \mathrm{g} / \mathrm{mL}$ against Bacillus subtilis and Escherichia coli.

The medicine granisetron containing the 9-azabicyclo[3.3.1]nonane moiety is a serotonin $5-\mathrm{HT}_{3}$ receptor antagonist [20] (Figure 1). This drug is used for preventing postoperative nausea and vomiting.

Novel polycationic materials based on 9-thia-, 9-aza-, and 9-selena[3.3.1]bicyclononanes have been synthesized and proposed as DNA-transfecting polymers [21-23]. An important desirable feature of DNA-transfecting polymers is the ability to degrade into non-toxic components after cellular uptake of a DNA-polymer complex. Cationic polymers composed of repeating units of 9-thia-, 9-aza-, and 9-selena[3.3.1]bicyclononanes have been found to show high transfection efficacy in a galactosidase assay.

Polymers and resins containing the 9-thiabicyclo[3.3.1]nonane and 9-selenabicyclo[3.3.1] nonane units have been applied for preparation of materials with high refractive in$\operatorname{dex}[24,25]$.

Derivatives of 9-azabicyclo[3.3.1]nonane have been proposed as CXCR6 receptor inhibitors [26] and JAK kinase inhibitors [27] (Figure 1).

The JAK kinase inhibitors are relatively new drugs exhibiting significant therapeutic advances. JAK kinase inhibitors are a type of medication that functions by inhibiting the activity of one or more of the Janus kinase family of enzymes. The JAK kinase inhibitors may have therapeutic application in the treatment of cancer, inflammatory diseases and various autoimmune diseases [27].

Selenium dichloride and dibromide were first involved in the synthesis of organoselenium compounds in $2003[28,29]$. The reaction of selenium dihalides with dimethyldiethynylsilane led to 3,6-dihalo-4,4-dimethyl-1,4-selenasilafulvenes [28]. Currently organic synthesis based on selenium dihalides is an intensively developing area of research [30-39]. Annulation reactions of selenium dihalides with unsaturated arenes gave various condensed heterocyclic compounds [40-44]. The addition of selenium dihalides to alkenes and alkynes afforded bis(2-haloalkyl) selenides [45,46] and bis(2-halovinyl) selenides in high yields [47-52]. Novel heterocycles [53-62] have been obtained by reactions of selenium dihalides with divinyl chalcogenides [63-65].

Extending our studies of the reactions of selenium dihalides with linear dienic compounds $[53-62,66]$, we explored their addition to cyclodienes. The reaction of selenium dihalides with cis,cis-1,5-cyclooctadiene occurred as transannular addition affording 2,6dihalo-9-selenabicyclo[3.3.1]nonanes in almost quantitative yields (Scheme 1) [16,67,68].

We studied nucleophilic substitution reaction of bromine in compound 2 by pyridine [69,70]. Dipiridinium salt, 2,6-dipyridiumyl-9-selenabicyclo[3.3.1]nonane dibromide, was obtained in near quantitative yield [69,70]. The biological activity of this compound as a medicine for metabolic correction in the vaccination process and its effect on immunogenesis were studied [69]. It was found that this compound considerably diminished the pathological effect caused by the action of the tularemia vaccine in experimental animals and significantly reduced the reactogenicity of the brucellosis vaccine. Based on these results, 2,6-dipyridiumyl-9-selenabicyclo[3.3.1]nonane dibromide was proposed as a promising drug for metabolic correction in the vaccination process [69]. 
<smiles></smiles>

Polymer with antimicrobial activity<smiles>COc1cc(C(=O)NC2CC3CCC(C2)N3CC(=O)Nc2ccccc2Cl)cc(OC)c1OC</smiles>

CXCR6 inhibitor<smiles>C1CCN(C2CCC3SC2CC3N2CCCCC2)CC1</smiles>

Antiinflammatory activity<smiles>CN1C2CCC1CC(NC(=O)c1nn(C)c3ccccc13)C2</smiles>

Granisetron<smiles>CCOS(=O)(=O)NC1C2CCC1CC(N(C)c1nc(CO)c(F)c(Nc3cc(C)[nH]n3)n1)C2</smiles>

JAK kinase inhibitor

(serotonin $5-\mathrm{HT}_{3}$ receptor antagonist)

Figure 1. Known biologically active derivatives of 9-thiabicyclo[3.3.1]nonane [18,19] and 9-azabicyclo[3.3.1]nonane [20,26,27].

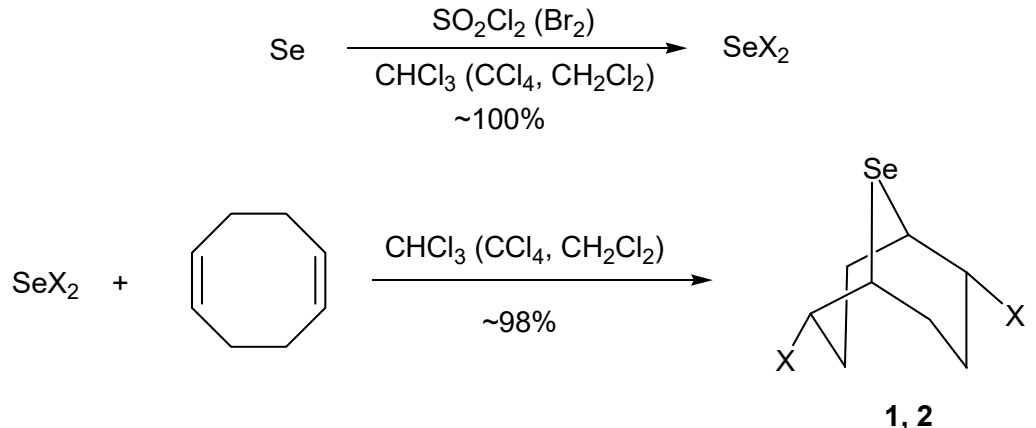

$\mathrm{X}=\mathrm{Cl}(\mathbf{1}), \mathrm{Br}(2)$

Scheme 1. Transannular addition of selenium dihalides to cis,cis-1,5-cyclooctadiene affording 2,6dihalo-9-selenabicyclo[3.3.1]nonanes $(\mathbf{1}, \mathbf{2})$.

\section{Results and Discussion}

Nucleophilic substitution reactions of bromine in compound 2 by sulfur-centered nucleophiles have not been studied and not a single representative of 2,6-disulfanyl-9selenabicyclo[3.3.1]nonanes has been described in the literature.

The efficient synthesis of a new family of 2,6-diorganylsulfanyl-9-selenabicyclo[3.3.1] nonanes has been developed in the present work (Figure 2). Theoretically, these compounds can be obtained by nucleophilic substitution reactions of bromine in compound 2 by organylthiols. However, we found a more efficient approach to 2,6-diorganylsulfanyl-9selenabicyclo[3.3.1]nonanes, which includes the preparation of bis-isothiouronium salt from compound 2 and thiourea. This approach opens up more synthetic possibilities and allows obtaining not only nucleophilic substitution products but also products of nucleophilic addition to a triple bond. 


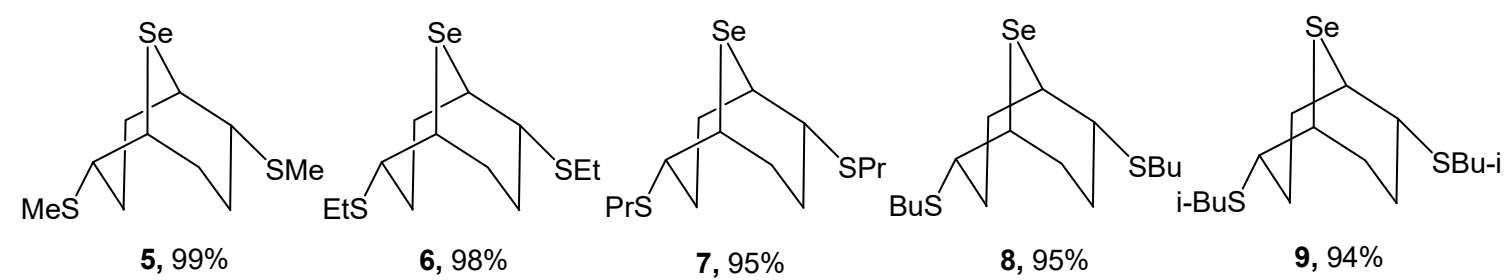<smiles>CC(C)SC1CCC2C(SC(C)C)C3CC1[Se]C23</smiles>

$10,90 \%$<smiles>c1ccc(CSC2CCC3[Se]C4C2CCC4C3SCc2ccccc2)cc1</smiles>

$11,92 \%$<smiles>Fc1ccc(CSC2CCC3CCCC2[Se]C3SCc2ccc(F)cc2)cc1</smiles>

$12,90 \%$<smiles>C=CCSC1CCC2CCCC1[Se]C2SCC=C</smiles>

$13,96 \%$<smiles>C=C(Cl)CSC1CCC2[Se]C1CCC2SCC(=C)Cl</smiles>

$14,94 \%$<smiles>C=C(C)CSC1CCC2CCC(SCC(=C)C)C1[Se]2</smiles>

$15,91 \%$<smiles>C(=C/c1ccccc1)\CSC1CCC2CC(SC/C=C/c3ccccc3)C3CC3C1C2</smiles>

$16,90 \%$<smiles>COC(=O)/C=C\SC1CCC2CCCC1[Se]2</smiles>

$17,84 \%$<smiles>CCOC(=O)/C=C\SC1CCC2CCCC1C2S/C=C\C(=O)OCC</smiles>

$18,81 \%$

Figure 2. A new family of compounds, 2,6-disulfanyl-9-selenabicyclo[3.3.1]nonane derivatives.

Bis-isothiouronium salt 3 was prepared in 95\% yield by the reaction of thiourea with compound 2 in acetonitrile under reflux. Bis-isothiouronium salt 3 precipitated under the reaction conditions and can be easily isolated (Scheme 2).<smiles>BrC1CCC2CCC(Br)C1O2</smiles>

2

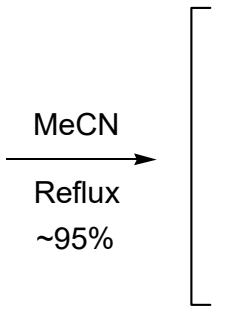<smiles>N=C(N)SC1CCC2CCCC1[Se]2</smiles>

3

Scheme 2. Synthesis of bis-isothiouronium salt 3 by the reaction of compound 2 with thiourea in acetonitrile.

The action of alkalis on bis-isothiouronium salt 3 led to generation of 9-selenabicyclo[3.3.1] nonane-2,6-dithiolate anion 4, which was involved in nucleophilic substitution reactions with a variety of alkylating reagents (Scheme 3 ). The conditions for efficient synthesis of 2,6-dialkylsulfanyl-9-selenabicyclo[3.3.1]nonanes have been found. In a typical procedure, sodium hydroxide was added to a methanol or ethanol solution containing alkylating reagent (MeI, EtBr, PrBr, BuBr, i-BuBr). 


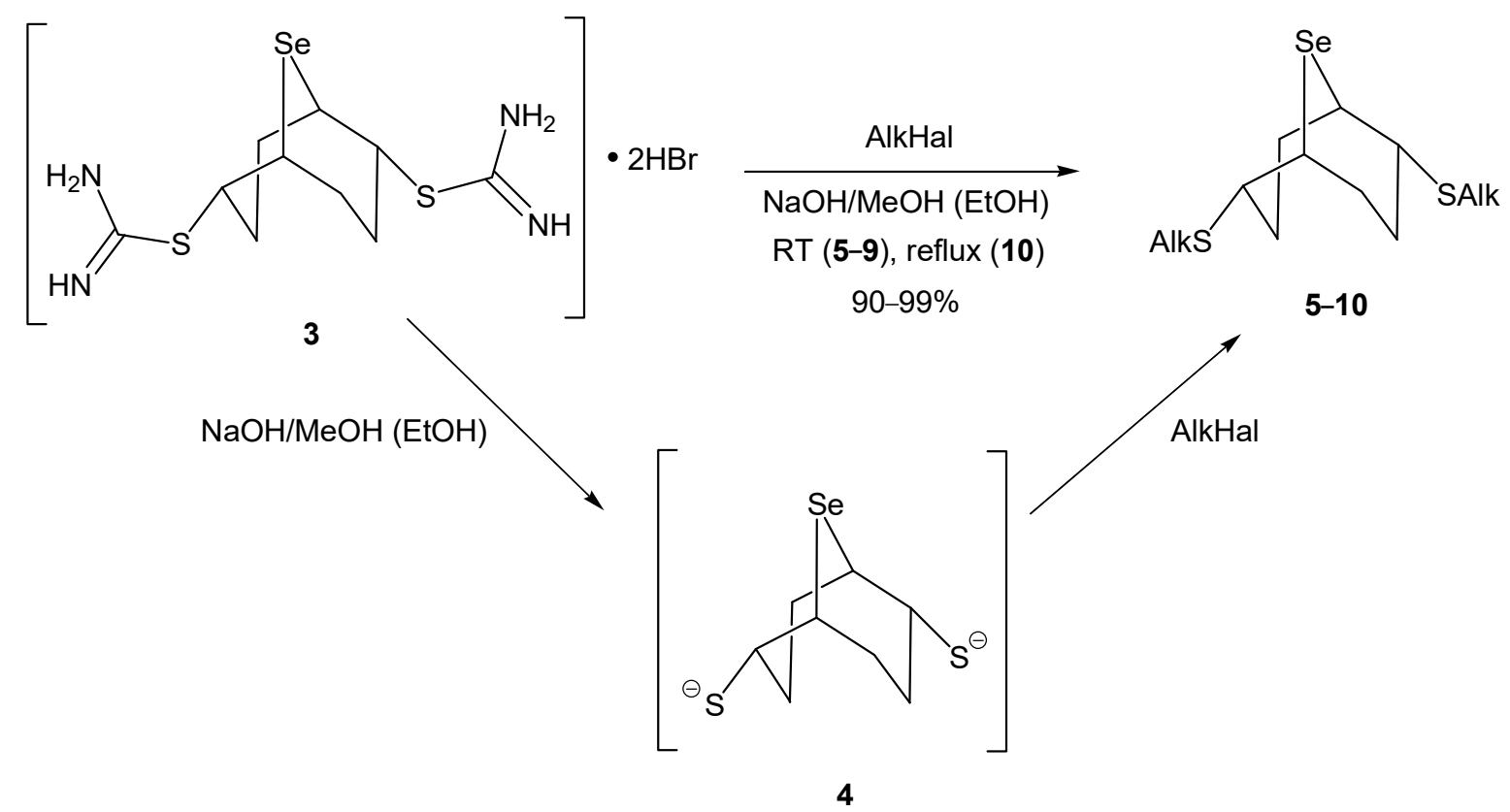

$\mathrm{Alk}=\mathrm{Me}, \mathrm{Hal}=\mathrm{I}(\mathbf{5}) ; \mathrm{Alk}=\mathrm{Et}, \mathrm{Hal}=\mathrm{Br}(\mathbf{6}) ; \mathrm{Alk}=\mathrm{Pr}, \mathrm{Hal}=\mathrm{Br}(7) ; \mathrm{Alk}=\mathrm{Bu}, \mathrm{Hal}=\mathrm{Br}(\mathbf{8})$; Alk $=\mathrm{i}-\mathrm{Bu}, \mathrm{Hal}=\mathrm{Br}(\mathbf{9}) ; \mathrm{Alk}=\mathrm{i}-\mathrm{Pr}, \mathrm{Hal}=\mathrm{Br}(\mathbf{1 0})$

Scheme 3. Synthesis of 2,6-di(alkylsulfanyl)-9-selenabicyclo[3.3.1]nonanes (5-10)) by nucleophilic substitution of alkyl halides by 9 -selenabicyclo[3.3.1]nonane-2,6-dithiolate anion generated from bis-isothiouronium salt 3.

The reaction proceeded under mild condition at room temperature in such "green solvents" as methanol or ethanol affording the target product 5-9 in 94-99\% yields without additional purification (Scheme 3).

In the case of the reaction of dithiolate anion 4 with isopropyl bromide at room temperature, the corresponding product $\mathbf{1 0}$ was formed only in 52\% yield. However, carrying out the process under reflux made it possible to accelerate this reaction and to obtain isopropyl derivative $\mathbf{1 0}$ in $90 \%$ yield after purification on a short column with silica gel (Scheme 3).

Although chlorine is usually displaced more slowly than bromine in nucleophilic substitution, the reactions of bis-isothiouronium salt 3 with benzyl and 4-fluorobenzyl chlorides proceeded smoothly at room temperature leading to 2,6-di(benzylsulfanyl)-9selenabicyclo[3.3.1]nonanes 11, 12 in 90-92\% yields (Scheme 4). It is worth noting that introduction of fluorine to organic molecules is usually favorable from the viewpoint of possible manifestation of biological activity and a number of modern important drugs contain the fluorine atom [71].

Allyl bromide easily reacted with bis-isothiouronium salt 3 at room temperature, leading to 2,6-di(allylsulfanyl)-9-selenabicyclo[3.3.1]nonane 13 in 96\% yields (Scheme 4). However, in the case of the reactions of bis-isothiouronium salt 3 with substituted allyl chlorides (3-chloro-2-methyl-1-propene, 2,3-dichloro-1-propene, E-3-chloro-1-propenylbenzene) under the same conditions at room temperature, corresponding products were obtained in $60-72 \%$ yields. In order to increase the yields of the products, the reactions of bisisothiouronium salt 3 with substituted allyl chlorides were carried out with heating $\left(50-60{ }^{\circ} \mathrm{C}\right)$. This made it possible to accelerate the reaction and to obtain compounds 14-16, which were isolated in $90-94 \%$ yields by purification on a short column with silica gel (Scheme 5). 
<smiles>N=C(N)SC1CCC2CCCC1[Se]2</smiles>

3<smiles>[R]c1ccc(CSCC2[Se]C3CC2CCC3SCc2ccc([R])cc2)cc1</smiles>

11,12

$\mathrm{R}=\mathrm{H}(\mathbf{1 1}), \mathrm{R}=\mathrm{F}(\mathbf{1 2})$

Scheme 4. Synthesis of 2,6-di(benzylsulfanyl)-9-selenabicyclo[3.3.1]nonanes (11, 12) from bis-isothiouronium salt 3 and benzyl halides.

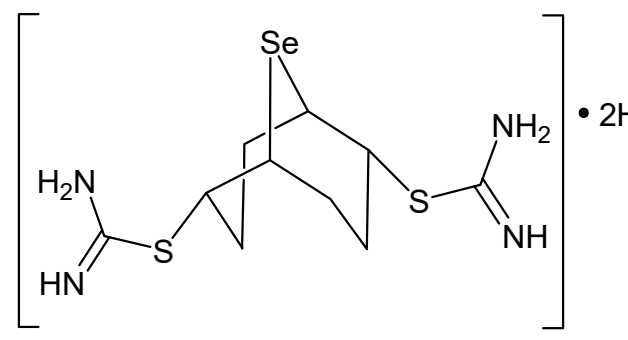

3

\section{$\underset{\mathrm{NaOH} / \mathrm{MeOH}(\mathrm{EtOH})}{\longrightarrow}$ \\ $\mathrm{Hal}=\mathrm{Br}(13), \mathrm{RT}$ \\ $\mathrm{Hal}=\mathrm{Cl}(\mathbf{1 4 - 1 6})$, reflux}

90-96\%<smiles>[R]C=C([X])CSC1CCC2CC1C(SCC([X])=C[R])C2[Se]</smiles>

13-16

$$
\mathrm{R}=\mathrm{H}, \mathrm{X}=\mathrm{H}(13) ; \mathrm{R}=\mathrm{H}, \mathrm{X}=\mathrm{Cl}(14) ; \mathrm{R}=\mathrm{H}, \mathrm{X}=\mathrm{Me}(15) ; \mathrm{R}=\mathrm{C}_{6} \mathrm{H}_{5} \mathrm{CH}_{2}, \mathrm{X}=\mathrm{H}(16)
$$

Scheme 5. Synthesis of 2,6-di(allylsulfanyl)-9-selenabicyclo[3.3.1]nonanes (13-16) from bis-isothiouronium salt 3 and allyl halides (allyl bromide, 2,3-dichloro-1-propene, 3-chloro-2-methyl-1-propene, E-3-chloro-1-propenylbenzene).

Finally, we realized the addition of 9-selenabicyclo[3.3.1]nonane-2,6-dithiolate anion to activated triple bond of alkyl propiolates. The conditions for efficient regio- and stereoselective reaction of bis-isothiouronium salt 3 with alkyl propiolates were established.

We found that it is advisable to carry out the reaction of bis-isothiouronium salt 3 with methyl propiolate in methanol and the process with ethyl propiolate advantageously to conduct in ethanol. Otherwise, the formation of some by-products derived from the interconversion of methyl and ethyl esters (the transesterification reaction in the presence of bases). Besides, the amount of alkali should be reduced by 2 times in comparison with the previous conditions for nucleophilic substitution reactions.

Thus, the reaction of nucleophilic addition of 9-selenabicyclo[3.3.1]nonane-2,6-dithiolate anion 4 to methyl and ethyl propiolates proceeded in a regio- and stereoselective manner, affording 2,6-di(vinylsulfanyl)-9-selenabicyclo[3.3.1]nonanes $\mathbf{1 7}$ (a ratio of Z/E isomers $\sim 17: 1$ ) in $84 \%$ yield and 18 (a ratio of $Z / E$ isomers $~ 11: 1$ ) in 81\% yield (Scheme 6). 


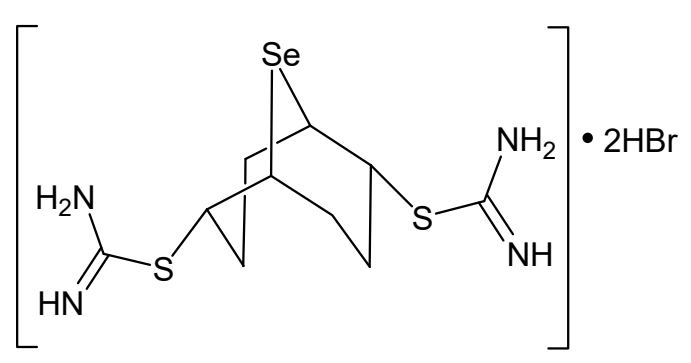

3

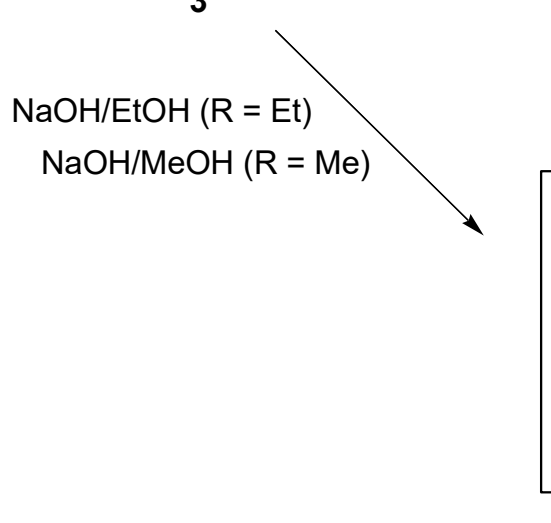

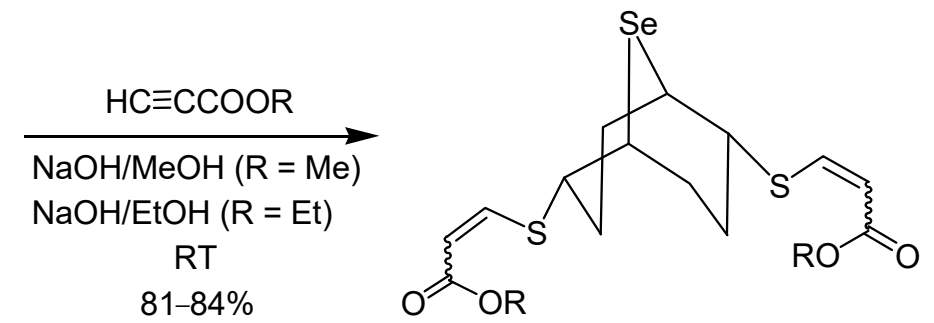

17, Z/E = $17: 1$

$18, \mathrm{Z} / \mathrm{E}=11: 1$

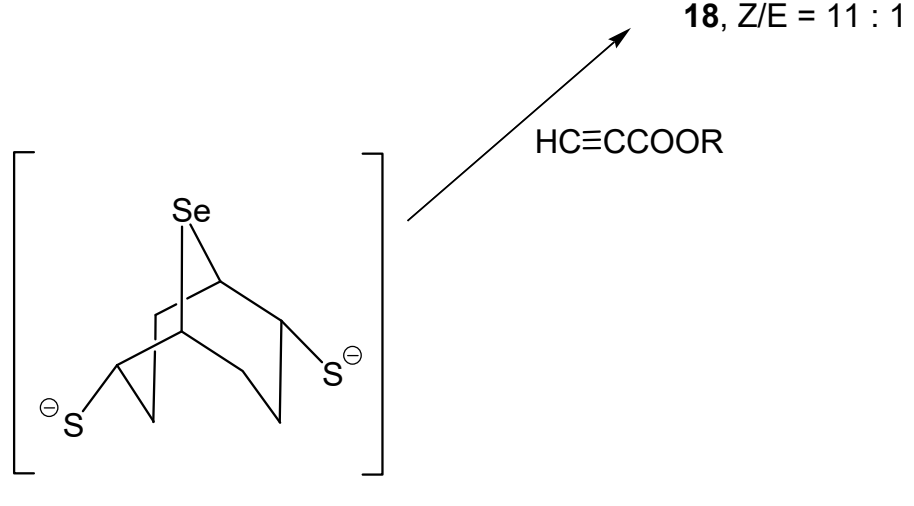

4

$\mathrm{R}=\mathrm{Me}(\mathbf{1 7}) ; \mathrm{R}=\mathrm{Et}(\mathbf{1 8})$

Scheme 6. Synthesis of 2,6-di(vinylsulfanyl)-9-selenabicyclo[3.3.1]nonanes $(\mathbf{1 7}, \mathbf{1 8})$ by nucleophilic addition of 9-selenabicyclo [3.3.1]nonane-2,6-dithiolate anion to alkyl propiolates.

The obtained products represent a new family of compounds, 2,6-disulfanyl-9-selenabicyclo [3.3.1]nonane derivatives (Figure 2), with promising biological activity.

The structural assignments of the synthesized compounds were made using ${ }^{1} \mathrm{H}$ and ${ }^{13} \mathrm{C}-\mathrm{NMR}$ spectroscopy and confirmed by elemental analysis. The signals of the CHSe group in ${ }^{13} \mathrm{C}$ NMR spectra of compounds 5-18 manifested themselves in the region 29.2$30.5 \mathrm{ppm}\left({ }^{1} J_{\mathrm{C}-\mathrm{Se}}=51.5-54.3 \mathrm{~Hz}\right)$. Stereoconfiguration of the vinyl group in compounds 17 and 18 was assigned based on the values of proton spin-spin coupling constants $\left({ }^{3} J_{\mathrm{H}-\mathrm{H}}\right)$, which are 10.0-10.2 Hz for (Z)-isomers and 15.2-15.3 Hz for (E)-isomers.

\section{Experimental Section}

\subsection{General Information}

The ${ }^{1} \mathrm{H}(400.1 \mathrm{MHz})$ and ${ }^{13} \mathrm{C}(100.6 \mathrm{MHz}) \mathrm{NMR}$ spectra (see Supplementary Materials) were recorded on a Bruker DPX-400 spectrometer (Bruker BioSpin GmbH, Rheinstetten, Germany) in $\mathrm{CDCl}_{3}$ (compounds 5-18) and $d_{6}$-DMSO (compounds 3 ) solutions and referred to the residual solvent peaks of $\mathrm{CDCl}_{3}\left(\delta=7.27\right.$ and $77.16 \mathrm{ppm}$ in ${ }^{1} \mathrm{H}$ - and ${ }^{13} \mathrm{C}-\mathrm{NMR}$, respectively) or $d_{6}$-DMSO ( $\delta=2.50$ and $39.5 \mathrm{ppm}$ in ${ }^{1} \mathrm{H}$ - and ${ }^{13} \mathrm{C}-\mathrm{NMR}$, respectively). Elemental analysis was performed on a Thermo Scientific Flash 2000 Elemental Analyzer (Thermo Fisher Scientific Inc., Milan, Italy). Melting points were determined on a Kofler Hot-Stage Microscope PolyTherm A apparatus (Wagner \& Munz GmbH, Munich, Germany). The organic solvents were dried and distilled according to standard procedures. Silica gel (Alfa Aesar, 0.06-0.20 mm (70-230 mesh) was used for column chromatography.

\subsection{Synthesis of Bis-Isothiouronium Salt 3}

2,6-Bis[amino(iminio)methylsulfanyl]-9-selenabicyclo[3.3.1]nonane dibromide (3). Thiourea $(1.52 \mathrm{~g}, 2 \mathrm{mmol})$ was added to a solution of compound 2 ( $3 \mathrm{~g}, 0.865 \mathrm{mmol})$ in acetonitrile $(120 \mathrm{~mL})$. The mixture was stirred at room temperature for $2 \mathrm{~h}$ and then heated under reflux with stirring for $5 \mathrm{~h}$. The formation of white precipitate was observed. Precipitated product 
was filtered, washed with cold hexane and dried in vacuum, giving bis-isothiouronium salt 3 ( $4.10 \mathrm{~g}, 95 \%$ yield) as a white powder; $\mathrm{mp} 219-220^{\circ} \mathrm{C}$.

${ }^{1} \mathrm{H}-\mathrm{NMR}$ (400 MHz, $d_{6}$-DMSO): 2.03-2.17 (m, 4H, $\left.\mathrm{CH}_{2} \mathrm{CHS}, \mathrm{CH}_{2} \mathrm{CHSe}\right), 2.32-2.41(\mathrm{~m}$, $\left.2 \mathrm{H}, \mathrm{CH}_{2} \mathrm{CHS}\right), 2.55-2.62\left(\mathrm{~m}, 2 \mathrm{H}, \mathrm{CH}_{2} \mathrm{CHSe}\right), 3.15-3.19(\mathrm{~m}, 2 \mathrm{H}, \mathrm{CHS}), 4.70-4.76(\mathrm{~m}, 2 \mathrm{H}$, CHSe), 9.02-9.30 (m, 8H, CN $\left.\mathrm{CH}_{4}\right) .{ }^{13} \mathrm{C}-\mathrm{NMR}\left(100 \mathrm{MHz}, d_{6}\right.$-DMSO): $27.9\left(\underline{\mathrm{CH}}_{2} \mathrm{CHSe}\right), 28.5$ ( $\left.\mathrm{CH}_{2} \mathrm{CHS}\right), 29.1$ (CHSe), $49.3(\mathrm{CHS}), 167.7(\mathrm{C}=\mathrm{N})$.

Anal. calcd for $\mathrm{C}_{10} \mathrm{H}_{20} \mathrm{~N}_{4} \mathrm{~S}_{2} \mathrm{Br}_{2} \mathrm{Se}$ (499.19): C 24.06, $\mathrm{H}$ 4.04, N 11.22, S 12.85, Br 32.01, Se 15.82\%. Found: C 23.91, H 3.99, N 11.20, S 12.80, Br 32.43, Se 15.98\%.

\subsection{Synthesis of Compounds $\mathbf{5 - 1 8}$}

2,6-Bis(methylsulfanyl)-9-selenabicyclo[3.3.1]nonane (5). A solution of methyl iodide $(0.26 \mathrm{~g}, 1.8 \mathrm{mmol})$ in ethanol $(1 \mathrm{~mL})$ was added to a solution of bis-isothiouronium salt $3(0.35 \mathrm{~g}, 0.7 \mathrm{mmol})$ in ethanol $(4 \mathrm{~mL})$. Then a solution of sodium hydroxide $(80 \%, 0.2 \mathrm{~g}$, $4 \mathrm{mmol}$ ) in ethanol $(3 \mathrm{~mL})$ was added dropwise to the reaction mixture. The mixture was stirred for $8 \mathrm{~h}$ at room temperature. Methylene chloride $(15 \mathrm{~mL})$ and cold water $(15 \mathrm{~mL})$ were added to the reaction mixture. The mixture was transferred to a separatory funnel and the organic layer was separated. The mixture was additionally extracted with methylene chloride $(2 \times 10 \mathrm{~mL})$, the organic phase was dried over $\mathrm{CaCl}_{2}$ and the solvent was removed by a rotary evaporator. The residue was dried in vacuum, giving product $5(0.195 \mathrm{~g}, 99 \%$ yield) as a white powder; $\mathrm{mp} 64-65^{\circ} \mathrm{C}$.

${ }^{1} \mathrm{H}$ NMR $\left(400 \mathrm{MHz}, \mathrm{CDCl}_{3}\right): 1.73-1.84\left(\mathrm{~m}, 2 \mathrm{H}, \mathrm{CH}_{2} \mathrm{CHS}\right), 1.93-2.02$ (m, 2H, $\left.\mathrm{CH}_{2} \mathrm{CHSe}\right)$, $2.03\left(\mathrm{~s}, 6 \mathrm{H}, \mathrm{CH}_{3}\right), 2.12-2.22\left(\mathrm{~m}, 2 \mathrm{H}, \mathrm{CH}_{2} \mathrm{CHS}\right), 2.64-2.73\left(\mathrm{~m}, 2 \mathrm{H}, \mathrm{CH}_{2} \mathrm{CHSe}\right), 2.97-3.02(\mathrm{~m}$, 2H, CHS), 3.47-3.54 (m, 2H, CHSe). ${ }^{13} \mathrm{C}$ NMR (100 MHz, $\left.\mathrm{CDCl}_{3}\right): 14.25\left(\mathrm{CH}_{3}\right), 28.8$ $\left(\mathrm{CH}_{2} \mathrm{CHSe}\right), 29.2\left(\mathrm{CHSe},{ }^{1} J_{\mathrm{Se}-\mathrm{C}}=51.5 \mathrm{~Hz}\right), 29.4\left(\mathrm{CH}_{2} \mathrm{CHS}\right), 48.3(\mathrm{CHS})$.

Anal. calcd for $\mathrm{C}_{10} \mathrm{H}_{18} \mathrm{~S}_{2} \mathrm{Se}$ (281.34): C $42.69, \mathrm{H}$ 6.45, S 22.79, Se 28.07\%. Found: C 42.91, H 6.46, S 22.98, Se 28.47\%.

2,6-Bis(ethylsulfanyl)-9-selenabicyclo[3.3.1]nonane (6). A solution of ethyl bromide (0.28 g, $2.6 \mathrm{mmol})$ in methanol $(1 \mathrm{~mL})$ was added to a solution of bis-isothiouronium salt $3(0.43 \mathrm{~g}$, $0.86 \mathrm{mmol})$ in methanol $(5 \mathrm{~mL})$. Then, a solution of sodium hydroxide $(80 \%, 0.25 \mathrm{~g}, 5 \mathrm{mmol})$ in methanol $(4 \mathrm{~mL})$ was added dropwise to the reaction mixture. The mixture was stirred overnight $(14 \mathrm{~h})$ at room temperature. Methylene chloride $(20 \mathrm{~mL})$ and cold water $(20 \mathrm{~mL})$ were added to the reaction mixture. The mixture was transferred to a separatory funnel and the organic layer was separated. The mixture was additionally extracted with methylene chloride $(2 \times 10 \mathrm{~mL})$, the organic phase was dried over $\mathrm{CaCl}_{2}$ and the solvent was removed by a rotary evaporator. The residue was dried in vacuum, giving product $6(0.26 \mathrm{~g}, 98 \%$ yield) as a white powder; $\mathrm{mp} 59-60{ }^{\circ} \mathrm{C}$.

${ }^{1} \mathrm{H}$ NMR $\left(400 \mathrm{MHz}, \mathrm{CDCl}_{3}\right): 1.15\left(\mathrm{t}, 6 \mathrm{H}, \mathrm{CH}_{3},{ }^{3} \mathrm{~J}_{\mathrm{H}-\mathrm{H}}=7.4 \mathrm{~Hz}\right), 1.76-1.88(\mathrm{~m}, 2 \mathrm{H}$, $\left.\mathrm{C}_{2} \mathrm{CHS}\right), 1.91-1.98$ (m, 2H, $\left.\underline{\mathrm{H}}_{2} \mathrm{CHSe}\right), 2.12-2.20$ (m, 2H, $\left.\mathrm{CH}_{2} \mathrm{CHS}\right), 2.41-2.53(\mathrm{~m}, 4 \mathrm{H}$, $\left.\mathrm{SCH}_{2} \mathrm{CH}_{3}\right), 2.64-2.73\left(\mathrm{~m}, 2 \mathrm{H}, \mathrm{CH}_{2} \mathrm{CHSe}\right), 2.93-2.97$ (m, 2H, CHS), 3.56-3.62 (m, 2H, CHSe). ${ }^{13} \mathrm{C}$ NMR $\left(100 \mathrm{MHz}, \mathrm{CDCl}_{3}\right): 14.9\left(\mathrm{CH}_{3}\right), 24.8\left(\mathrm{CH}_{2} \mathrm{CH}_{3}\right), 29.0\left(\mathrm{CH}_{2} \mathrm{CHSe}\right), 29.9\left(\underline{\mathrm{CH}}_{2} \mathrm{CHS}\right)$, 30.0 (CHSe, $\left.{ }^{1} J_{\mathrm{Se}-\mathrm{C}}=52.7 \mathrm{~Hz}\right), 48.3$ (CHS).

Anal. calcd for $\mathrm{C}_{12} \mathrm{H}_{22} \mathrm{~S}_{2} \mathrm{Se}$ (309.39): C 46.58, H 7.17, S 20.73, Se 25.52\%. Found: C 46.31, H 7.15, S 20.88, Se 25.86\%.

2,6-Bis(propylsulfanyl)-9-selenabicyclo[3.3.1]nonane (7) was obtained as a colourless viscous oil ( $0.276 \mathrm{~g}, 95 \%$ yield) from bis-isothiouronium salt $3(0.43 \mathrm{~g}, 0.86 \mathrm{mmol})$, propyl bromide $(0.32 \mathrm{~g}, 2.6 \mathrm{mmol})$ and sodium hydroxide $(80 \%, 0.25 \mathrm{~g}, 5 \mathrm{mmol})$ in methanol under the same conditions as compound 6 .

${ }^{1} \mathrm{H}$ NMR $\left(400 \mathrm{MHz}, \mathrm{CDCl}_{3}\right): 0.89\left(\mathrm{t}, 6 \mathrm{H}, \mathrm{CH}_{3},{ }^{3} \mathrm{~J}_{\mathrm{H}-\mathrm{H}}=7.4 \mathrm{~Hz}\right), 1.43-1.55(\mathrm{~m}, 4 \mathrm{H}$, $\left.\mathrm{C}_{2} \mathrm{CH}_{3}\right), 1.76-1.88$ (m, 2H, $\left.\mathrm{CH}_{2} \mathrm{CHSe}\right), 1.91-1.99$ (m, 2H, $\left.\mathrm{CH}_{2} \mathrm{CHS}\right), 2.09-2.22(\mathrm{~m}, 2 \mathrm{H}$, $\left.\mathrm{CH}_{2} \mathrm{CHSe}\right), 2.36-2.49\left(\mathrm{~m}, 4 \mathrm{H}, \mathrm{CH}_{2} \mathrm{~S}\right), 2.65-2.74\left(\mathrm{~m}, 2 \mathrm{H}, \mathrm{CH}_{2} \mathrm{CHS}\right), 2.92-2.97(\mathrm{~m}, 2 \mathrm{H}, \mathrm{CHS})$, 3.54-3.58 (m, 2H, CHSe). ${ }^{13} \mathrm{C}$ NMR (100 MHz, $\left.\mathrm{CDCl}_{3}\right): 13.4\left(\mathrm{CH}_{3}\right), 23.1\left(\mathrm{CH}_{2} \mathrm{CH}_{3}\right), 29.0$ $\left(\mathrm{CH}_{2} \mathrm{CHSe}\right), 30.0\left(\mathrm{CH}_{2} \mathrm{CHS}\right), 30.1\left(\mathrm{CHSe},{ }^{1} J_{\mathrm{Se}-\mathrm{C}}=52.6 \mathrm{~Hz}\right), 33.0\left(\mathrm{CH}_{2} \mathrm{~S}\right), 48.8(\mathrm{CHS})$.

Anal. calcd for $\mathrm{C}_{14} \mathrm{H}_{26} \mathrm{~S}_{2} \mathrm{Se}$ (337.45): $\mathrm{C} 49.83, \mathrm{H}$ 7.77, $\mathrm{S} 19.00$, Se $23.40 \%$. Found: $\mathrm{C}$ 49.65, H 7.63, S 19.34, Se 23.86\%. 
2,6-Bis(butylsulfanyl)-9-selenabicyclo[3.3.1]nonane (8) was obtained as a colourless viscous oil $(0.299 \mathrm{~g}, 95 \%$ yield $)$ from bis-isothiouronium salt $3(0.43 \mathrm{~g}, 0.86 \mathrm{mmol})$, butyl bromide $(0.35 \mathrm{~g}, 2.6 \mathrm{mmol})$ and sodium hydroxide $(80 \%, 0.25 \mathrm{~g}, 5 \mathrm{mmol})$ in methanol under the same conditions as compound 6.

${ }^{1} \mathrm{H}$ NMR $\left(400 \mathrm{MHz}, \mathrm{CDCl}_{3}\right): 0.82\left(\mathrm{t}, 6 \mathrm{H}, \mathrm{CH}_{3},{ }^{3} \mathrm{~J}_{\mathrm{H}-\mathrm{H}}=7.2 \mathrm{~Hz}\right), 1.27-1.36(\mathrm{~m}, 4 \mathrm{H}$, $\left.\mathrm{CH}_{2} \mathrm{CH}_{3}\right), 1.43-1.50$ (m, 4H, $\left.\underline{\mathrm{CH}}_{2} \mathrm{CH}_{2} \mathrm{~S}\right), 1.74-1.88$ (m, 2H, $\left.\underline{\mathrm{H}}_{2} \mathrm{CHSe}\right), 1.92-1.99$ (m, 2H, $\left.\mathrm{CH}_{2} \mathrm{CHS}\right), 2.12-2.22\left(\mathrm{~m}, 2 \mathrm{H}, \mathrm{CH}_{2} \mathrm{CHSe}\right), 2.40-2.52\left(\mathrm{~m}, 4 \mathrm{H}, \mathrm{CH}_{2} \mathrm{~S}\right), 2.66-2.74(\mathrm{~m}, 2 \mathrm{H}$, $\left.\mathrm{CH}_{2} \mathrm{CHS}\right), 2.94-2.99$ (m, 2H, CHS), 3.54-3.59 (m, 2H, CHSe). $\left.{ }^{13} \mathrm{C} \mathrm{NMR} \mathrm{(100} \mathrm{MHz,} \mathrm{CDCl}_{3}\right)$ : $13.7\left(\mathrm{CH}_{3}\right), 22.0\left(\underline{\mathrm{CH}}_{2} \mathrm{CH}_{3}\right), 29.1\left(\underline{\mathrm{CH}}_{2} \mathrm{CHSe}\right), 30.1\left(\underline{\mathrm{CH}}_{2} \mathrm{CHS}\right), 30.1\left(\mathrm{CHSe},{ }^{1} J_{\mathrm{Se}-\mathrm{C}}=52.6 \mathrm{~Hz}\right)$, $30.7\left(\mathrm{CH}_{2} \mathrm{CH}_{2} \mathrm{~S}\right), 32.0\left(\mathrm{CH}_{2} \mathrm{~S}\right), 48.9$ (CHS).

Anal. calcd for $\mathrm{C}_{16} \mathrm{H}_{30} \mathrm{~S}_{2}$ Se (365.50): C 52.58, H 8.27, S 17.55, Se 21.60\%. Found: C 52.75, H 8.19, S 17.74, Se 21.42\%.

2,6-Bis(isobutylsulfanyl)-9-selenabicyclo[3.3.1]nonane (9) was obtained as a colourless viscous oil ( $0.296 \mathrm{~g}, 94 \%$ yield $)$ from bis-isothiouronium salt $3(0.43 \mathrm{~g}, 0.86 \mathrm{mmol})$, isobutyl bromide $(0.35 \mathrm{~g}, 2.6 \mathrm{mmol})$ and sodium hydroxide $(80 \%, 0.25 \mathrm{~g}, 5 \mathrm{mmol})$ in methanol under the same conditions as compound 6.

${ }^{1} \mathrm{H}$ NMR (400 MHz, $\left.\mathrm{CDCl}_{3}\right): 0.98\left(\mathrm{~d}, 12 \mathrm{H}, \mathrm{CH}_{3},{ }^{3} \mathrm{~J}_{\mathrm{H}-\mathrm{H}}=6.7 \mathrm{~Hz}\right), 1.70-1.81(\mathrm{~m}, 2 \mathrm{H}$, C $\underline{\mathrm{H}}_{2} \mathrm{CHS}$ ), $1.86-1.97$ (m, 2H, $\mathrm{CH}_{2} \mathrm{CHSe}$ ), 2.02-2.10 (m, 2H, $\left.\mathrm{CH}_{2} \mathrm{CHS}\right), 2.20-2.30$ (m, 2H, $\left.\mathrm{CH}_{2} \mathrm{CHSe}\right), 2.36-2.48\left(\mathrm{~m}, 4 \mathrm{H} \mathrm{SCH}_{2}\right), 2.76-2.82$ (m, 2H, $\left.\underline{\mathrm{HCH}}_{2} \mathrm{~S}\right), 3.02-3.05$ (m, 2H, CHS), 3.59-3.65 (m, 2H, CHSe). $\left.{ }^{13} \mathrm{C} \mathrm{NMR} \mathrm{(100} \mathrm{MHz,} \mathrm{CDCl}_{3}\right): 22.1\left(\mathrm{CH}_{3}\right), 22.3\left(\mathrm{CH}_{3}\right), 29.0$ $\left(\mathrm{CHCH}_{3}\right), 29.3\left(\underline{\mathrm{CH}}_{2} \mathrm{CHSe}\right), 30.2\left(\underline{\mathrm{CH}}_{2} \mathrm{CHS}\right), 30.3\left(\mathrm{CHSe},{ }^{1} J_{\mathrm{Se}-\mathrm{C}}=52.1 \mathrm{~Hz}\right), 40.4\left(\mathrm{CH}_{2} \mathrm{~S}\right)$, 48.3 (CHS).

Anal. calcd for $\mathrm{C}_{16} \mathrm{H}_{30} \mathrm{~S}_{2}$ Se (365.50): C 52.58, H 8.27, S 17.55, Se 21.60\%. Found: C 52.34, H 8.15, S 17.41, Se 21.84\%.

2,6-Bis(isopropylsulfanyl)-9-selenabicyclo[3.3.1]nonane (10). A solution of isopropyl bromide $(0.32 \mathrm{~g}, 2.6 \mathrm{mmol})$ in methanol $(1 \mathrm{~mL})$ was added to a solution of compound $3(0.43 \mathrm{~g}$, $0.86 \mathrm{mmol})$ in methanol $(5 \mathrm{~mL})$. Then a solution of sodium hydroxide $(80 \%, 0.25 \mathrm{~g}, 5 \mathrm{mmol})$ in methanol $(4 \mathrm{~mL})$ was added dropwise and the mixture was refluxed for $3 \mathrm{~h}$. Methylene chloride $(20 \mathrm{~mL})$ and cold water $(20 \mathrm{~mL})$ were added to the reaction mixture. The mixture was transferred to a separatory funnel and the organic layer was separated. The mixture was additionally extracted with methylene chloride $(2 \times 10 \mathrm{~mL})$, the organic phase was dried over $\mathrm{CaCl}_{2}$ and the solvent was removed by a rotary evaporator. The residue was subjected to column chromatography on silica gel (eluent: hexane, then hexane/chloroform 1:10). Compound $10(0.262 \mathrm{~g}, 90 \%$ yield $)$ was isolated as a colourless viscous oil.

${ }^{1} \mathrm{H}$ NMR $\left(400 \mathrm{MHz} \mathrm{CDCl}_{3}\right): 1.19\left(\mathrm{~d}, 12 \mathrm{H}, \mathrm{CH}_{3},{ }^{3} \mathrm{~J}_{\mathrm{H}-\mathrm{H}}=6.7 \mathrm{~Hz}\right), 1.78-1.91(\mathrm{~m}, 2 \mathrm{H}$, $\left.\mathrm{CH}_{2} \mathrm{CHS}\right), 1.98-2.18\left(\mathrm{~m}, 4 \mathrm{H}, \mathrm{CH}_{2} \mathrm{CHSe}, \mathrm{CH}_{2} \mathrm{CHS}\right), 2.41-2.53\left(\mathrm{~m}, 4 \mathrm{H}, \mathrm{SCH}_{2} \mathrm{CH}_{3}\right), 2.65-2.74$ (m, 2H, $\left.\mathrm{CH}_{2} \mathrm{CHSe}\right), 2.78-2.99$ (m, 4H, CHS, $\mathrm{CH}_{3} \mathrm{CHS}$ ), 3.56-3.62 (m, 2H, CHSe).

${ }^{13} \mathrm{C} \mathrm{NMR}\left(100 \mathrm{MHz}, \mathrm{CDCl}_{3}\right): 23.6,23.9\left(\mathrm{CH}_{3}\right), 29.3\left(\mathrm{CH}_{2} \mathrm{CHSe}\right), 30.3\left(\mathrm{CH}_{2} \mathrm{CHS}\right), 30.5$ (CHSe, $\left.{ }^{1} J_{\text {Se-C }}=53.6 \mathrm{~Hz}\right), 33.9$ ( $\left.\mathrm{CH}_{3} \underline{\mathrm{CHS}}\right), 47.4$ (CHS).

2,6-Bis(benzylsulfanyl)-9-selenabicyclo[3.3.1]nonane (11) was obtained from bis-isothiouronium salt $3(0.43 \mathrm{~g}, 0.86 \mathrm{mmol})$, benzyl bromide $(0.41 \mathrm{~g}, 2.4 \mathrm{mmol})$ and sodium hydroxide $(80 \%$, $0.25 \mathrm{~g}, 5 \mathrm{mmol}$ ) in methanol under the same conditions as compound 6 . The product was purified by column chromatography on silica gel (eluent: hexane, then hexane/chloroform 1:10). Compound $11(0.342 \mathrm{~g}, 92 \%$ yield $)$ was isolated as a white powder; $\mathrm{mp} 71-72{ }^{\circ} \mathrm{C}$.

${ }^{1} \mathrm{H}$ NMR (400 MHz, CDCl 3 ): 1.81-1.97 (m, 4H, $\left.\underline{\mathrm{CH}}_{2} \mathrm{CHS}, \mathrm{CH}_{2} \mathrm{CHSe}\right), 2.09-2.18$ (m, 2H, $\mathrm{CH}_{2} \mathrm{CHS}$ ), 2.67-2.74 (m, 2H, $\left.\underline{\mathrm{CH}}_{2} \mathrm{CHSe}\right), 2.88-2.94$ (m, 2H, CHS), 3.50-3.56 (m, 2H, CHSe), 3.65-3.72 (m, 4H, SC $\left.\underline{\mathrm{H}}_{2} \mathrm{Ar}\right), 7.15-7.23\left(\mathrm{~m}, 2 \mathrm{H}, \mathrm{CH}_{\mathrm{Ar}}\right), 7.21-7.32\left(\mathrm{~m}, 8 \mathrm{H}, \mathrm{CH}_{\mathrm{Ar}}\right) .{ }^{13} \mathrm{C} \mathrm{NMR}$ (100 MHz, CDCl $\left.\mathrm{M}_{3}\right): 29.2$ ( $\left.\mathrm{CH}_{2} \mathrm{CHSe}\right), 29.7\left(\mathrm{CH}_{2} \mathrm{CHS}\right), 29.8\left(\mathrm{CHSe},{ }^{1} J_{\mathrm{Se}-\mathrm{C}}=52.9 \mathrm{~Hz}\right), 35.6$ $\left(\mathrm{ArCH}_{2} \mathrm{~S}\right), 48.4(\mathrm{CHS}), 127.1\left(\mathrm{C}_{\mathrm{Ar}}\right), 128.6\left(\mathrm{CH}_{\mathrm{Ar}}\right), 128.7\left(\mathrm{CH}_{\mathrm{Ar}}\right), 138.4\left(\mathrm{C}_{\mathrm{Ar}}\right)$.

Anal. calcd for $\mathrm{C}_{22} \mathrm{H}_{26} \mathrm{~S}_{2}$ Se (433.53): C 60.95, H 6.04, S 14.79, Se 18.21\%. Found: C 60.56, H 6.01, S 14.96, Se 18.54\%.

2,6-Bis(4-fluorobenzylsulfanyl)-9-selenabicyclo[3.3.1]nonane (12) was obtained from bisisothiouronium salt $3(0.43 \mathrm{~g}, 0.86 \mathrm{mmol})$, 4-fluorobenzyl bromide $(0.456 \mathrm{~g}, 2.4 \mathrm{mmol})$ and sodium hydroxide $(80 \%, 0.25 \mathrm{~g}, 5 \mathrm{mmol})$ in methanol under the same conditions as 
compound 6. The product was purified by column chromatography on silica gel (eluent: hexane, then hexane/chloroform 1:10). Compound 12 (0.363 g, 90\% yield) was isolated as a white powder; $\mathrm{mp} 84-85^{\circ} \mathrm{C}$.

${ }^{1} \mathrm{H}$ NMR (400 MHz, $\mathrm{CDCl}_{3}$ ): 1.84-2.00 (m, 4H, $\left.\mathrm{CH}_{2} \mathrm{CHSe}, \mathrm{CH}_{2} \mathrm{CHS}\right), 2.14-2.22$ (m, 2H, $\mathrm{CH}_{2} \mathrm{CHS}$ ), 2.66-2.73 (m, 2H, $\left.\mathrm{CH}_{2} \mathrm{CHSe}\right), 2.91-2.96$ (m, 2H, CHS), 3.52-3.58 (m, 2H, CHSe), 3.67-3.74 (m, 4H, $\left.\mathrm{CH}_{2} \mathrm{~S}\right), 6.96-7.00\left(\mathrm{~m}, 4 \mathrm{H}, \mathrm{C}_{\mathrm{Ar}}\right), 7.4-7.27\left(\mathrm{~m}, 4 \mathrm{H}, \mathrm{CH}_{\mathrm{Ar}}\right) .{ }^{13} \mathrm{C}$ $\mathrm{NMR}\left(100 \mathrm{MHz}, \mathrm{CDCl}_{3}\right): 29.2$ ( $\left.\mathrm{CH}_{2} \mathrm{CHSe}\right), 29.7$ ( $\left.\mathrm{CH}_{2} \mathrm{CHS}\right), 29.7\left(\mathrm{CHSe},{ }^{1} J_{\mathrm{Se}-\mathrm{C}}=53.0 \mathrm{~Hz}\right)$, $34.8\left(\mathrm{CH}_{2} \mathrm{~S}\right), 48.5$ (CHS), 115.4, $115.6\left(\mathrm{HC}_{\mathrm{Ar}}\right), 130.2\left(\mathrm{HC}_{\mathrm{Ar}}\right), 134.1\left(\mathrm{CH}_{2} \mathrm{C}_{\mathrm{Ar}}\right), 160.7,163.1$ $\left(\mathrm{FC}_{\mathrm{Ar}},{ }^{1} J_{\mathrm{F}-\mathrm{C}}=245.7 \mathrm{~Hz}\right)$.

Anal. calcd for $\mathrm{C}_{22} \mathrm{H}_{24} \mathrm{~F}_{2} \mathrm{~S}_{2} \mathrm{Se}$ (469.51): C 56.28, H 5.15, F 8.09, S 13.66, Se 16.82\%. Found: C 55.98, H 5.06, S 13.76, Se 17.02\%.

2,6-Bis(allylsulfanyl)-9-selenabicyclo[3.3.1]nonane (13) was obtained as a colourless viscous oil (0.302 $\mathrm{g}, 96 \%$ yield) from bis-isothiouronium salt 3 (0.43 g, $0.86 \mathrm{mmol})$, allyl bromide $(0.315 \mathrm{~g}, 2.6 \mathrm{mmol})$ and sodium hydroxide $(80 \%, 0.25 \mathrm{~g}, 5 \mathrm{mmol})$ in methanol under the same conditions as compound 6.

${ }^{1} \mathrm{H}$ NMR (400 MHz, $\mathrm{CDCl}_{3}$ ): 1.79-1.97 (m, 4H, $\left.\underline{\mathrm{H}}_{2} \mathrm{CHSe}, \mathrm{CH}_{2} \mathrm{CHS}\right), 2.11-2.22$ (m, $2 \mathrm{H}$, $\mathrm{CH}_{2} \mathrm{CHS}$ ), 2.65-2.74 (m, 2H, $\left.\mathrm{CH}_{2} \mathrm{CHSe}\right), 2.87-2.97$ (m, 2H, CHS), 3.03-3.15 (m, 4H, $\mathrm{CH}_{2} \mathrm{~S}$ ), 3.49-3.56 (m, 2H, CHSe), 4.98-5.05 (dd, 4H, $\left.\mathrm{CH}_{2}=\mathrm{CH},{ }^{3} J_{\mathrm{H}-\mathrm{H}}=9.9 \mathrm{~Hz},{ }^{3} J_{\mathrm{H}-\mathrm{H}}=16.8 \mathrm{~Hz}\right)$, 5.67-5.78 (m, 2H, $\left.\mathrm{CH}=\mathrm{CH}_{2}\right) .{ }^{13} \mathrm{C} \mathrm{NMR}\left(100 \mathrm{MHz}, \mathrm{CDCl}_{3}\right): 29.1\left(\mathrm{CH}_{2} \mathrm{CHSe}\right), 29.6(\mathrm{CHSe}$, $\left.{ }^{1} J_{\mathrm{Se}-\mathrm{C}}=52.9 \mathrm{~Hz}\right), 29.6\left(\underline{\mathrm{CH}}_{2} \mathrm{CHS}\right), 33.9\left(\mathrm{CH}_{2} \mathrm{~S}\right), 48.8(\mathrm{CHS}), 116.7\left(\underline{\mathrm{CH}}_{2}=\mathrm{CH}\right), 134.4\left(\underline{\mathrm{CH}}=\mathrm{CH}_{2}\right)$. Anal. calcd for $\mathrm{C}_{14} \mathrm{H}_{22} \mathrm{~S}_{2}$ Se (333.41): C 50.43, H 6.65, S 19.23, Se 23.68\%. Found: C 50.03 , H 6.55, S 19.48, Se 23.99\%.

2,6-Bis(2-chloro-2-propenylsulfanyl)-9-selenabicyclo[3.3.1]nonane (14). A solution of 2,3dichloro-1-propene $(0.289 \mathrm{~g}, 2.6 \mathrm{mmol})$ in methanol $(1 \mathrm{~mL})$ was added to a solution of compound $3(0.43 \mathrm{~g}, 0.86 \mathrm{mmol})$ in methanol $(5 \mathrm{~mL})$. Then, a solution of sodium hydroxide $(80 \%, 0.25 \mathrm{~g}, 5 \mathrm{mmol})$ in methanol $(4 \mathrm{~mL})$ was added dropwise and the mixture was heated at $50-60{ }^{\circ} \mathrm{C}$ with stirring for $7 \mathrm{~h}$. Methylene chloride $(20 \mathrm{~mL})$ and cold water $(20 \mathrm{~mL})$ were added to the reaction mixture. The mixture was transferred to a separatory funnel and the organic layer was separated. The mixture was additionally extracted with methylene chloride $(2 \times 10 \mathrm{~mL})$, the organic phase was dried over $\mathrm{CaCl}_{2}$ and the solvent was removed by a rotary evaporator. The residue was subjected to column chromatography on silica gel (eluent: hexane, then hexane/chloroform 1:10). Compound 14 (0.325 g, 94\% yield) was isolated as a colourless viscous oil.

${ }^{1} \mathrm{H}$ NMR (400 MHz, $\left.\mathrm{CDCl}_{3}\right)$ : 1.89-2.01 (m, 2H, $\left.\mathrm{CH}_{2} \mathrm{CHSe}\right), 2.05-2.11$ (m, 2H, $\left.\mathrm{CH}_{2} \mathrm{CHS}\right)$, 2.24-2.33 (m, 2H, $\underline{\mathrm{CH}}_{2} \mathrm{CHSe}$ ), 2.74-2.83 (m, 2H, $\left.\underline{\mathrm{CH}}_{2} \mathrm{CHS}\right), 3.00-3.06$ (m, 2H, CHS), 3.32-3.38 $\left(\mathrm{m}, 2 \mathrm{H}, \mathrm{CH}_{2} \mathrm{~S}\right), 3.42-3.48\left(\mathrm{~m}, 2 \mathrm{H}, \mathrm{CH}_{2} \mathrm{~S}\right), 3.65-3.69(\mathrm{~m}, 2 \mathrm{H}, \mathrm{CHSe}), 5.29$ (s, 2H, $\left.\mathrm{H}_{2} \mathrm{C}=\mathrm{CCl}\right)$, $5.38\left(\mathrm{~s}, 2 \mathrm{H}, \mathrm{H}_{2} \mathrm{C}=\mathrm{CCl}\right) .{ }^{13} \mathrm{C}$ NMR $\left(100 \mathrm{MHz}, \mathrm{CDCl}_{3}\right): 29.4\left(\underline{\mathrm{CH}}_{2} \mathrm{CHSe}\right), 29.8\left(\underline{\mathrm{CH}_{2} \mathrm{CHS}}\right)$, 29.9 (CHSe), $39.2\left(\mathrm{CH}_{2} \mathrm{~S}\right), 48.9$ (CHS), $114.5\left(\mathrm{H}_{2} \mathrm{C}=\mathrm{CCl}\right), 139.1\left(\mathrm{ClC}=\mathrm{CH}_{2}\right)$. Anal. calcd for $\mathrm{C}_{14} \mathrm{H}_{20} \mathrm{~S}_{2} \mathrm{Cl}_{2}$ Se (402.30): C 41.80, H 5.01, S 15.94, Cl 17.63, Se 19.63\%. Found: C 41.66, H 4.98, S 16.07, Cl 17.51, Se 19.92\%.

2,6-Bis(2-methyl-2-propenylsulfanyl)-9-selenabicyclo[3.3.1]nonane (15) was obtained from bis-isothiouronium salt $3(0.43 \mathrm{~g}, 0.86 \mathrm{mmol}), 3$-chloro-2-methyl-1-propene, (0.313 g, $2.5 \mathrm{mmol}$ ) and sodium hydroxide $(80 \%, 0.25 \mathrm{~g}, 5 \mathrm{mmol})$ in methanol under the same conditions as compound 14. The product was purified by column chromatography on silica gel (eluent: hexane, then hexane/chloroform 1:10). Compound 15 (0.283 g, 91\% yield) was isolated as a white powder; $\mathrm{mp} 52-53{ }^{\circ} \mathrm{C}$.

${ }^{1} \mathrm{H}$ NMR (400 MHz, $\left.\mathrm{CDCl}_{3}\right): 1.80\left(\mathrm{~s}, 6 \mathrm{H}, \mathrm{CH}_{3}\right), 1.86-2.02\left(\mathrm{~m}, 4 \mathrm{H}, \mathrm{CH}_{2} \mathrm{CHS}, \mathrm{CH}_{2} \mathrm{CHSe}\right)$, 2.17-2.29 (m, 2H, $\mathrm{CH}_{2} \mathrm{CHS}$ ), 2.71-2.78 (m, 2H, $\underline{\mathrm{CH}}_{2} \mathrm{CHSe}$ ), 2.96-3.00 (m, 2H, CHS), 3.02-3.11 (m, 2H, $\left.\mathrm{CH}_{2} \mathrm{~S}\right), 3.14-3.21\left(\mathrm{~m}, 2 \mathrm{H}, \mathrm{CH}_{2} \mathrm{~S}\right), 3.50-3.54$ (m, 2H, CHSe), 4.79-4.83 (m, 4H, $\mathrm{CH}_{2}=\mathrm{C}$ ). ${ }^{13} \mathrm{C} \mathrm{NMR}\left(100 \mathrm{MHz}, \mathrm{CDCl}_{3}\right): 20.8\left(\mathrm{CH}_{3}\right), 29.4\left(\mathrm{CH}_{2} \mathrm{CHSe}\right), 29.8\left(\mathrm{CHSe}^{1} \mathrm{~J}_{\mathrm{Se}-\mathrm{C}}=52.8 \mathrm{~Hz}\right)$, $29.8\left(\underline{\mathrm{CH}}_{2} \mathrm{CHS}\right), 38.7\left(\mathrm{CH}_{2} \mathrm{~S}\right), 48.0(\mathrm{CHS}), 113.4\left(\mathrm{H}_{2} \underline{\mathrm{C}}=\mathrm{C}\right), 141.6\left(\underline{\mathrm{C}}=\mathrm{CH}_{2}\right)$.

Anal. calcd for $\mathrm{C}_{16} \mathrm{H}_{26} \mathrm{~S}_{2}$ Se (361.47): C 53.16, $\mathrm{H} 7.25, \mathrm{~S} 17.74$, Se 21.84\%. Found: C 52.98, H 7.22, S 17.89, Se 22.04\%. 
2,6-Bis[(E)-3-phenyl-2-propenyl)sulfanyl]-9-selenabicyclo[3.3.1]nonane (16) was obtained from bis-isothiouronium salt $3(0.43 \mathrm{~g}, 0.86 \mathrm{mmol})$, (E)-3-chloro-1-propenylbenzene $(0.365 \mathrm{~g}$, $2.4 \mathrm{mmol})$ and sodium hydroxide $(80 \%, 0.25 \mathrm{~g}, 5 \mathrm{mmol})$ in methanol under the same conditions as compound 14. The product was purified by column chromatography on silica gel (eluent: hexane, then hexane/chloroform 1:10). Compound 16 (0.376 g, 90\% yield) was isolated as a colourless viscous oil.

${ }^{1} \mathrm{H}$ NMR (400 MHz, $\mathrm{CDCl}_{3}$ ): 1.99-2.19 (m, 4H, $\left.\mathrm{CH}_{2} \mathrm{CHS}, \mathrm{CH}_{2} \mathrm{CHSe}\right), 2.32-2.41$ (m, 2H, $\mathrm{CH}_{2} \mathrm{CHS}$ ), 2.88-2.95 (m, 2H, $\left.\underline{\mathrm{CH}}_{2} \mathrm{CHSe}\right), 3.10-3.16$ (m, 2H, CHS), 3.34-3.44 ( $\left.\mathrm{CH}_{2} \mathrm{~S}\right), 3.77-3.81$ $(\mathrm{m}, 2 \mathrm{H}, \mathrm{CHSe}), 6.22-6.34\left(\mathrm{~m}, 2 \mathrm{H}, \mathrm{CH}=\mathrm{CHC}_{\mathrm{Ar}}\right), 6.52\left(\mathrm{~d}, 2 \mathrm{H}, \mathrm{PhC} \underline{\mathrm{H}}=\mathrm{CH},{ }^{3} J_{\mathrm{H}-\mathrm{H}}=16.0 \mathrm{~Hz}\right)$, 7.31-7.35 (m, 2H, $\left.\mathrm{CH}_{\mathrm{Ar}}\right), 7.38-7.43\left(\mathrm{~m}, 4 \mathrm{H}, \mathrm{CH}_{\mathrm{Ar}}\right), 7.45-7.48\left(\mathrm{~m}, 4 \mathrm{H}, \mathrm{CH}_{\mathrm{Ar}}\right) .{ }^{13} \mathrm{C} \mathrm{NMR}$ (100 MHz, CDCl 3$): 29.2\left(\mathrm{CH}_{2} \mathrm{CHSe}\right), 29.7\left(\mathrm{CHSe}^{1} \mathrm{~J}_{\mathrm{Se}-\mathrm{C}}=52.8 \mathrm{~Hz}\right), 29.7\left(\mathrm{CH}_{2} \mathrm{CHS}\right), 33.4$ $\left(\mathrm{CH}_{2} \mathrm{~S}\right), 47.7(\mathrm{CHS}), 125.9\left(\mathrm{C}_{\mathrm{Ar}}\right), 126.2\left(\mathrm{CH}_{\mathrm{Ar}}\right), 127.4\left(\mathrm{C}_{\mathrm{Vin}}\right), 128.4\left(\mathrm{CH}_{\mathrm{Ar}}\right), 131.8\left(\mathrm{C}_{\text {Vin }}\right), 136.5$ $\left(\mathrm{C}_{\mathrm{Ar}}\right)$.

Anal. calcd for $\mathrm{C}_{26} \mathrm{H}_{30} \mathrm{~S}_{2} \mathrm{Se}$ (485.61): C 64.31, H 6.23, S 13.21, Se 16.26\%. Found: C 64.03, H 6.14, S 13.34, Se 16.43\%.

2,6-Bis(3-methoxy-3-oxo-1-propenylsulfanyl)-9-selenabicyclo[3.3.1]nonane (17) (a ratio of $\mathrm{Z} / \mathrm{E}$ isomers $\sim 17: 1)$. A solution of methyl propiolate $(0.2 \mathrm{~g}, 2.28 \mathrm{mmol})$ in methanol $(1 \mathrm{~mL})$ was added to a solution of compound $3(0.35 \mathrm{~g}, 0.7 \mathrm{mmol})$ in methanol $(5 \mathrm{~mL})$. Then, a solution of sodium hydroxide $(80 \%, 0.1 \mathrm{~g}, 2 \mathrm{mmol})$ in methanol $(3 \mathrm{~mL})$ was added dropwise and the mixture was stirred overnight $(15 \mathrm{~h})$. Methylene chloride $(15 \mathrm{~mL})$ and cold water $(15 \mathrm{~mL})$ were added to the reaction mixture. The mixture was transferred to a separatory funnel and the organic layer was separated. The mixture was additionally extracted with methylene chloride $(2 \times 10 \mathrm{~mL})$, the organic phase was dried over $\mathrm{CaCl}_{2}$ and the solvent was removed by a rotary evaporator. The residue was subjected to column chromatography on silica gel (eluent: hexane, then hexane/chloroform 1:10). Compound 17 (0.248 g, 84\% yield) was isolated as a white powder; $\mathrm{mp} 165-166^{\circ} \mathrm{C}$.

(Z)-isomer (Z-17). ${ }^{1} \mathrm{H}$ NMR (400 MHz, $\left.\mathrm{CDCl}_{3}\right)$ : 1.94-2.05 (m, 2H, $\left.\mathrm{CH}_{2} \mathrm{CHS}\right), 2.08-2.15$ (m, 2H, $\underline{\mathrm{CH}}_{2} \mathrm{CHSe}$ ), 2.21-2.30 (m, 2H, $\mathrm{CH}_{2} \mathrm{CHS}$ ), 2.70-2.77 (m, 2H, $\mathrm{CH}_{2} \mathrm{CHSe}$ ), 3.04-3.10 (m, 2H, $\left.\mathrm{CH}_{2} \mathrm{CHS}\right), 3.68\left(\mathrm{~s}, 6 \mathrm{H}, \mathrm{CH}_{3}\right), 3.80-3.87$ (m, 2H, CHSe), $5.82(\mathrm{~d}, 2 \mathrm{H}, \underline{\mathrm{HC}}=\mathrm{CHS}$, $\left.{ }^{3} \mathrm{~J}_{\mathrm{H}-\mathrm{H}}=10.0 \mathrm{~Hz}\right), 7.10\left(\mathrm{~d}, 2 \mathrm{H}, \mathrm{HC}=\mathrm{C} \underline{\mathrm{HS}},{ }^{3} \mathrm{~J}_{\mathrm{H}-\mathrm{H}}=10.0 \mathrm{~Hz}\right) .{ }^{13} \mathrm{C} \mathrm{NMR}\left(100 \mathrm{MHz}, \mathrm{CDCl}_{3}\right): 28.7$ ( $\left.\underline{\mathrm{CH}}_{2} \mathrm{CHSe}\right), 29.4$ ( $\left.\underline{\mathrm{CH}}_{2} \mathrm{CHS}\right), 30.3\left(\overline{\mathrm{CHSe}},{ }^{1} \mathrm{~J}_{\mathrm{Se}-\mathrm{C}}=53.8 \mathrm{~Hz}\right), 51.3(\mathrm{CHS}), 53.3\left(\mathrm{CH}_{3}\right), 113.1$ $(\mathrm{HC}=\mathrm{CHS}), 147.6$ (HC= $\underline{\mathrm{CHS}}), 166.8$ (COO).

(E)-isomer (E-17). ${ }^{1} \mathrm{H}$ NMR (400 MHz, $\left.\mathrm{CDCl}_{3}\right)$ : 1.94-2.05 (m, 2H, $\left.\mathrm{CH}_{2} \mathrm{CHS}\right), 2.08-2.15$ (m, 2H, $\mathrm{CH}_{2} \mathrm{CHSe}$ ), 2.21-2.30 (m, 2H, $\mathrm{CH}_{2} \mathrm{CHS}$ ), 2.70-2.77 (m, 2H, $\mathrm{CH}_{2} \mathrm{CHSe}$ ), 3.04-3.10 (m, 2H, $\left.\mathrm{CH}_{2} \mathrm{C} \underline{\mathrm{HS}}\right), 3.64\left(\mathrm{~s}, 6 \mathrm{H}, \mathrm{CH}_{3}\right), 3.80-3.87$ (m, 2H, CHSe), 5.78 (d, 2H, $\underline{\mathrm{HC}}=\mathrm{CHS}$, $\left.{ }^{3} J_{\mathrm{H}-\mathrm{H}}=15.4 \mathrm{~Hz}\right), 7.59\left(\mathrm{~d}, 2 \mathrm{H}, \mathrm{HC}=\mathrm{C} \underline{\mathrm{HS}},{ }^{3} \mathrm{~J}_{\mathrm{H}-\mathrm{H}}=15.4 \mathrm{~Hz}\right) .{ }^{13} \mathrm{C} \mathrm{NMR}\left(100 \mathrm{MHz}, \mathrm{CDCl}_{3}\right)$ : 28.9 ( $\underline{\mathrm{CH}}_{2} \mathrm{CHSe}$ ), 29.0 ( $\left.\underline{\mathrm{CH}}_{2} \mathrm{CHS}\right), 29.3$ (CHSe), 50.38 (CHS), $53.4\left(\mathrm{CH}_{3}\right), 115.1$ (HC $\left.=\mathrm{CHS}\right)$, $145.3(\mathrm{HC}=\mathrm{CHS}), 165.5(\mathrm{COO})$.

Anal. calcd for $\mathrm{C}_{16} \mathrm{H}_{22} \mathrm{O}_{4} \mathrm{~S}_{2} \mathrm{Se}$ (421.43): C 45.60, H 5.26, O 15.19, S 15.22, Se 18.74\%. Found: C 45.34, H 5.12, S 15.34, Se 19.01\%.

2,6-Bis(3-ethoxy-3-oxo-1-propenylsulfanyl)-9-selenabicyclo[3.3.1]nonane (18) (a ratio of Z/E isomers $\sim 11: 1)$ was obtained from bis-isothiouronium salt $3(0.35 \mathrm{~g}, 0.7 \mathrm{mmol})$, ethyl propiolate $(0.22 \mathrm{~g}, 2.24 \mathrm{mmol})$ and sodium hydroxide $(80 \%, 0.1 \mathrm{~g}, 2 \mathrm{mmol})$ in methanol under the same conditions as compound 17. The product was purified by column chromatography on silica gel (eluent: hexane, then hexane/chloroform 1:10). Compound 18 (0.255 g, 81\% yield) was isolated as a white powder; $\mathrm{mp} 125-127^{\circ} \mathrm{C}$.

(Z)-isomer (Z-18). ${ }^{1} \mathrm{H}$ NMR (400 MHz, $\left.\mathrm{CDCl}_{3}\right)$ : 1.01-1.11 (s, 6H, $\left.\mathrm{CH}_{3},{ }^{3} \mathrm{~J}_{\mathrm{H}-\mathrm{H}}=7.1 \mathrm{~Hz}\right)$, 1.78-1.90 (m, 2H, C $\underline{\mathrm{H}}_{2} \mathrm{CHS}$ ), 1.93-2.00 (m, 2H, $\left.\mathrm{CH}_{2} \mathrm{CHSe}\right), 2.06-2.17$ (m, 2H, $\mathrm{CH}_{2} \mathrm{CHS}$ ), 2.56-2.63 (m, 2H, $\left.\mathrm{CH}_{2} \mathrm{CHSe}\right), 2.92-2.99$ (m, 2H, $\left.\mathrm{CH}_{2} \mathrm{CHS}\right), 3.56-3.64$ (m, 2H, CHSe), 3.93$4.02\left(\mathrm{~m}, 4 \mathrm{H}, \mathrm{CH}_{3} \mathrm{CH}_{2}\right), 5.66\left(\mathrm{~d}, 2 \mathrm{H},(\mathrm{HC}=\mathrm{CHS}),{ }^{3} J_{\mathrm{H}-\mathrm{H}}=10.2 \mathrm{~Hz}\right), 7.00(\mathrm{~d}, 2 \mathrm{H},(\mathrm{HC}=\underline{\mathrm{CHS}})$, $\left.{ }^{3} \mathrm{~J}_{\mathrm{H}-\mathrm{H}}=10.2 \mathrm{~Hz}\right) .{ }^{13} \mathrm{C} \mathrm{NMR}\left(100 \mathrm{MHz}, \mathrm{CDCl}_{3}\right): 13.9\left(\mathrm{CH}_{3}\right), 28.3\left(\underline{\mathrm{CH}_{2} \mathrm{CHSe}}\right), 28.9\left(\underline{\mathrm{CH}}_{2} \mathrm{CHS}\right)$, $29.8\left(\mathrm{CHSe}^{1} \mathrm{~J}_{\mathrm{Se}-\mathrm{C}}=54.3 \mathrm{~Hz}\right), 52.7(\mathrm{CHS}), 59.6\left(\mathrm{CH}_{2} \mathrm{O}\right), 112.9(\mathrm{SHC}=\underline{\mathrm{CH}}), 147.0(\mathrm{HC}=\underline{\mathrm{CHS}})$, 165.9 (COO). 
(E)-isomer (E-18). ${ }^{1} \mathrm{H}$ NMR (400 MHz, $\left.\mathrm{CDCl}_{3}\right): 1.01-1.11\left(\mathrm{~s}, 6 \mathrm{H}, \mathrm{CH}_{3},{ }^{3} \mathrm{~J}_{\mathrm{H}-\mathrm{H}}=7.1 \mathrm{~Hz}\right)$ 1.78-1.90 (m, 2H, C $\left.\underline{\mathrm{H}}_{2} \mathrm{CHS}\right), 1.93-2.00$ (m, 2H, $\left.\mathrm{CH}_{2} \mathrm{CHSe}\right), 2.06-2.17$ (m, 2H, $\underline{\mathrm{H}}_{2} \mathrm{CHS}$ ), 2.47-2.53 (m, 2H, CH $\left.\mathrm{CH}_{2} \mathrm{CHSe}\right), 2.92-2.99$ (m, 2H, CH $\mathrm{CHS}_{2}, 3.56-3.64$ (m, 2H, CHSe), 3.93$4.02\left(\mathrm{~m}, 4 \mathrm{H}, \mathrm{CH}_{3} \overline{\mathrm{CH}}_{2}\right), 5.62\left(\mathrm{~d}, 2 \mathrm{H}, \underline{\mathrm{HC}}=\mathrm{CHS},{ }^{3} \mathrm{~J}_{\mathrm{H}-\mathrm{H}}=15.3 \mathrm{~Hz}\right), 7.44(\mathrm{~d}, 2 \mathrm{H}, \mathrm{HC}=\mathrm{C} \underline{\mathrm{HS}}$, $\left.{ }^{3} \mathrm{~J}_{\mathrm{H}-\mathrm{H}}=15.3 \mathrm{~Hz}\right) .{ }^{13} \overline{\mathrm{CNMR}}\left(100 \mathrm{MHz}, \mathrm{CDCl}_{3}\right): 13.9\left(\mathrm{CH}_{3}\right), 28.5\left(\underline{\mathrm{CH}_{2} \mathrm{CHSe}}\right), 28.6\left(\underline{\mathrm{CH}}_{2} \mathrm{CHS}\right)$, 29.1 (CHSe), 52.6 (CHS), 59.7 ( $\left.\mathrm{CH}_{2} \mathrm{O}\right), 114.9$ (HC=CHS), 144.6 (HC= $\left.\underline{\mathrm{CHS}}\right), 164.5$ (COO).

Anal. calcd for $\mathrm{C}_{18} \mathrm{H}_{26} \mathrm{O}_{4} \mathrm{~S}_{2} \mathrm{Se}$ (449.49): $\mathrm{C} 48.10, \mathrm{H} 5.83$, O 14.24, S 14.27, Se 17.57\%. Found: C 47.96, H 5.75, S 14.41, Se 17.72\%.

\section{Conclusions}

Bis-isothiouronium salt 3 was prepared in 95\% yield from thiourea and 2,6-dibromo-9selenabicyclo[3.3.1]nonane derived from the transannular addition of selenium dibromide to cis,cis-1,5-cyclooctadiene. Bis-isothiouronium salt 3 was served as valuable starting material for generation of 9-selenabicyclo[3.3.1]nonane-2,6-dithiolate anion. The latter was involved in nucleophilic substitution reactions with alkyl, benzyl and allyl halides, affording 2,6-disulfanyl-9-selenabicyclo[3.3.1]nonane derivatives 5-16 in 90-99\% yields.

The conditions for efficient nucleophilic addition of 9-selenabicyclo[3.3.1]nonane-2,6dithiolate anion to a triple bond of alkyl propiolates have been found. The reaction of bis-isothiouronium salt 3 with alkyl propiolates proceeded in a regio- and stereoselective manner affording 2,6-di(vinylsulfanyl)-9-selenabicyclo[3.3.1]nonanes 17 and 18 in 81-84\% yields. The obtained 2,6-disulfanyl-9-selenabicyclo[3.3.1]nonane derivatives are a new family of compounds with promising biological activity.

Supplementary Materials: The following are available online, the NMR spectra of the obtained compounds.

Author Contributions: Research experiments and spectral data processing: M.V.M.; methodology and the paper preparation: V.A.P.; conceptualization and data curation: S.V.A. All authors have read and agreed to the published version of the manuscript.

Funding: This research received no external funding.

Institutional Review Board Statement: Not applicable.

Informed Consent Statement: Not applicable.

Data Availability Statement: Data is available in this article and supplementary information.

Acknowledgments: The authors thank the Baikal Analytical Center SB RAS for providing the instrumental equipment for structural investigations.

Conflicts of Interest: The authors declare no conflict of interest.

Sample Availability: Samples of the compounds are not available from the authors.

\section{References}

1. Vitaku, E.; Smith, D.T.; Njardarson, J.T. Analysis of the Structural Diversity, Substitution Patterns, and Frequency of Nitrogen Heterocycles among U.S. FDA Approved Pharmaceuticals. J. Med. Chem. 2014, 57, 10257-10274. [CrossRef]

2. Gomtsyan, A. Heterocycles in drugs and drug discovery. Chem. Heterocycl. Compd. 2012, 48, 7-10. [CrossRef]

3. Mundy, B.P.; Ellerd, M.G.; Favaloro, F.G. Name Reactions and Reagents in Organic Synthesis, 2nd ed.; Wiley-Interscience: Hoboken, NJ, USA, 2005.

4. Seeman, J.I. Albert Padwa: A renaissance man of great passion and grit. Heterocycles 2012, 84, 63-73. [CrossRef]

5. Nogueira, C.W.; Zeni, G.; Rocha, J.B.T. Organoselenium and Organotellurium Compounds: Toxicology and Pharmacology. Chem. Rev. 2004, 104, 6255-6286. [CrossRef]

6. Ruberte, A.C.; Sanmartin, C.; Aydillo, C.; Sharma, A.K.; Plano, D. Development and Therapeutic Potential of Selenazo Compounds. J. Med. Chem. 2020, 63, 1473-1489. [CrossRef] [PubMed]

7. Yu, S.-C.; Kuhn, H.; Daniliuc, C.-G.; Ivanov, I.; Jones, P.G.; du Mont, W.-W. 5-Selenization of salicylic acid derivatives yielded isoform-specific 5-lipoxygenase inhibitors. Org. Biomol. Chem. 2010, 8, 828-834. [CrossRef]

8. Santi, C. (Ed.) Organoselenium Chemistry: Between Synthesis and Biochemistry; Bentham Science Publishers: Sharjah, United Arab Emirates, 2014; p. 563. 
9. Potapov, V.A. Organic diselenides, ditellurides, polyselenides and polytellurides. Synthesis and reactions. In Patai's Chemistry of Functional Groups. Organic Selenium and Tellurium Compounds; Rappoport, Z., Ed.; John Wiley and Sons: Chichester, UK, 2013; Volume 4, pp. 765-843.

10. Mugesh, G.; du Mont, W.W.; Sies, H. Chemistry of Biologically Important Synthetic Organoselenium Compounds. Chem. Rev. 2001, 101, 2125-2180. [CrossRef] [PubMed]

11. Tiekink, E.R.T. Therapeutic potential of selenium and tellurium compounds: Opportunities yet unrealized. Dalton Trans. 2012, 41, 6390-6395. [CrossRef]

12. Woollins, J.D.; Laitinen, R.S. (Eds.) Selenium and Tellurium Chemistry. From Small Molecules to Biomolecules and Materials; Springer: Heidelberg, Germany, 2011; p. 334.

13. Braga, A.L.; Rafique, J. Synthesis of biologically relevant small molecules containing selenium. Part B. Anti-infective and anticancer compounds. In Patai's Chemistry of Functional Groups. Organic Selenium and Tellurium Compounds; Rappoport, Z., Ed.; John Wiley and Sons: Chichester, UK, 2013; Volume 4, pp. 1053-1117.

14. Azad, G.K.; Tomar, R.S. Ebselen, a promising antioxidant drug: Mechanisms of action and targets of biological pathways. Mol. Biol. Rep. 2014, 41, 4865-4879. [CrossRef] [PubMed]

15. Lenardao, E.J.; Santi, C.; Sancineto, L. New Frontiers in Organoselenium Compounds; Springer International Publishing AG: Cham, Switzerland, 2018; 189p.

16. Accurso, A.A.; Cho, S.-H.; Amin, A.; Potapov, V.A.; Amosova, S.V.; Finn, M.G. Thia-, Aza-, and Selena[3.3.1]bicyclononane Dichlorides: Rates vs Internal Nucleophile in Anchimeric Assistance. J. Org. Chem. 2011, 76, 4392-4395. [CrossRef] [PubMed]

17. Finn, M.G.; Beveridge, J.M.; Geoghan, A.F.; Geng, Z. Antimicrobial coatings and methods of making and using thereof. WO Patent 2017210347. Chem. Abstrs. 2017, 168, 62266.

18. Tolstikov, G.A.; Krivonogov, V.P.; Galimov, B.I.; Lazareva, D.N.; Davydova, V.A.; Krivonogova, I.I.; Murinov, Y.I. Synthesis and antiinflammatory activity of 9-thiabicyclo[3.3.1]nonanes. Khimiko-Farmatsevticheskii Zhurnal. 1997, 31, 26-29. (In Russian) [CrossRef]

19. Geng, Z.; Finn, M.G. Thiabicyclononane-Based Antimicrobial Polycations. J. Am. Chem. Soc. 2017, 139, 15401-15406. [CrossRef]

20. Midani, D.; Parkman, H.P. Granisetron Transdermal System for Treatment of Symptoms of Gastroparesis: A Prescription Registry Study. J. Neurogastroenterol. Motility 2016, 22, 650-655. [CrossRef] [PubMed]

21. Geng, Z.; Garren, M.; Finn, M.G. Thiabicyclononane-Based Hyperbranched Polycations for Low-Dose Oligonucleotide Delivery. Chem. Mater. 2018, 30, 8164-8169. [CrossRef]

22. Geng, Z.; Finn, M.G. Fragmentable Polycationic Materials Based on Anchimeric Assistance. Chem. Mater. 2016, 28, 146-152. [CrossRef]

23. Accurso, A.A.; Baksh, M.M.; Cho, S.-H.; Potapov, V.A.; Amosova, S.V.; Finn, M.G. Thia-, aza-, and selena[3.3.1]bicyclononane polycationic materials: A new approach to transfecting polymers based on anchimeric assistance. Am. Chem. Soc. Div. Polym. Mater. Sci. Eng. 2009, 101, 1234-1235.

24. Cao, F.; Zhang, J.; Guo, L.; Yi, X.; Liu, Y. Polyurethane type resin with high refractive index and preparation method thereof. CN Patent 107118322. Chem. Abstrs. 2017, 168, 76844.

25. Okazaki, K.; Tanaka, M.; Honma, S.; Morijiri, H.; Kanemura, Y.; Kusumoto, M. Selenized polymers for transparent optical materials. WO Patent 9837117. Chem. Abstrs. 1998, 129, 231726.

26. Peddibhotla, S.; Hershberger, P.M.; Kirby, R.J.; Malany, S.; Smith, L.H.; Maloney, P.R.; Sessions, H.; Divlianska, D.; Pinkerton, A.B. Preparation of azabicyclononanes and diazabicyclononanes as CXCR6 inhibitors and methods of use. WO Patent 2021007208. Chem. Abstrs. 2021, 174, 310381.

27. Kozak, J.; Hudson, R.; Brandt, G.E.L.; Mckinnell, R.M.; Dabros, M.; Nzerem, J. Preparation of pyrimidine compound as JAK kinase inhibitor. WO Patent 2019084383. Chem. Abstrs. 2019, 170, 573104.

28. Potapov, V.A.; Amosova, S.V.; Belozerova, O.V.; Albanov, A.I.; Yarosh, O.G.; Voronkov, M.G. Synthesis of 3,6-dihalo-4,4-dimethyl1,4-selenasilafulvenes. Chem. Heterocycl. Compd. 2003, 39, 549-550. [CrossRef]

29. Potapov, V.A.; Amosova, S.V. New Methods for Preparation of Organoselenium and Organotellurium Compounds from Elemental Chalcogens. Russ. J. Org. Chem. 2003, 39, 1373-1380. [CrossRef]

30. Abakumov, G.A.; Piskunov, A.V.; Cherkasov, V.K.; Fedushkin, I.L.; Ananikov, V.P.; Eremin, D.B.; Gordeev, E.G.; Beletskaya, I.P.; Averin, A.D.; Bochkarev, M.N.; et al. Organoelement chemistry: Promising growth areas and challenges. Russ. Chem. Rev. 2018, 87, 393-507. [CrossRef]

31. Braverman, S.; Cherkinsky, M.; Kalendar, Y.; Jana, R.; Sprecher, M.; Goldberg, I. Synthesis of water-soluble vinyl selenides and their high glutathione peroxidase (GPx)-like antioxidant activity. Synthesis 2014, 46, 119-125. [CrossRef]

32. Sarbu, L.G.; Hopf, H.; Jones, P.G.; Birsa, L.M. Selenium halide-induced bridge formation in [2.2]paracyclophanes. Beilstein J. Org. Chem. 2014, 10, 2550-2555. [CrossRef]

33. Arsenyan, P. A simple method for the preparation of selenopheno[3,2- $b]$ and $[2,3-b]$ thiophenes. Tetrahedron Lett. 2014, 55, 2527-2529. [CrossRef]

34. Arsenyan, P.; Petrenko, A.; Belyakov, S. Improved conditions for the synthesis and transformations of aminomethyl selenophenothiophenes. Tetrahedron 2015, 71, 2226-2233. [CrossRef]

35. Musalov, M.V.; Musalova, M.V.; Potapov, V.A.; Albanov, A.I.; Amosova, S.V. Methoxyselenation of Cyclopentene with Selenium Dibromide. Russ. J. Org. Chem. 2015, 51, 1662-1663. [CrossRef] 
36. Potapov, V.A.; Musalov, M.V.; Kurkutov, E.O.; Musalova, M.V.; Albanov, A.I.; Amosova, S.V. Synthesis of New Functionalized Organoselenium Compounds by Heterocyclization of Selenium Dihalides with Pent-4-en-1-ol. Russ. J. Org. Chem. 2016, 52, 339-342. [CrossRef]

37. Volkova, Y.M.; Makarov, A.Y.; Zikirin, S.B.; Genaev, A.M.; Bagryanskaya, I.Y.; Zibarev, A.V. 3,1,2,4-Benzothiaselenadiazine and related heterocycles. Mendeleev Commun. 2017, 27, 19-22. [CrossRef]

38. Musalov, M.V.; Potapov, V.A. Selenium dihalides: New possibilities for the synthesis of selenium-containing heterocycles. Chem. Heterocycl. Comp. 2017, 53, 150-152. [CrossRef]

39. Potapov, V.A.; Musalov, M.V.; Musalova, M.V.; Amosova, S.V. Recent Advances in Organochalcogen Synthesis Based on Reactions of Chalcogen Halides with Alkynes and Alkenes. Curr. Org. Chem. 2016, 20, 136-145. [CrossRef]

40. Musalov, M.V.; Yakimov, V.A.; Potapov, V.A.; Amosova, S.V.; Borodina, T.N.; Zinchenko, S.V. A novel methodology for the synthesis of condensed selenium heterocycles based on the annulation and annulation-methoxylation reactions of selenium dihalides. New J. Chem. 2019, 43, 18476-18483. [CrossRef]

41. Musalov, M.V.; Potapov, V.A.; Amosova, S.V. Reaction of selenium dichloride with allyl phenyl ether. Russ. J. Org. Chem. 2011, 47, 948-949. [CrossRef]

42. Musalov, M.V.; Potapov, V.A.; Musalova, M.V.; Amosova, S.V. Annulation of phenyl propargyl ether with selenium dichloride. Russ. Chem. Bull. Int. Ed. 2010, 60, 767-768. [CrossRef]

43. Potapov, V.A.; Musalov, M.V.; Musalova, M.V.; Amosova, S.V. Regioselective Synthesis of Bis[(2,3-dihydro-1-benzofuran-2yl)methyl]selenide. Russ. J. Org. Chem. 2014, 50, 1702-1703.

44. Potapov, V.A.; Musalov, M.V.; Amosova, S.V. Reactions of selenium dichloride and dibromide with unsaturated ethers. Annulation of 2,3-dihydro-1,4-oxaselenine to the benzene ring. Tetrahedron Lett. 2011, 52, 4606-4610. [CrossRef]

45. Potapov, V.A.; Musalov, M.V.; Amosova, S.V.; Kurkutov, E.O. Reactions of Selenium Bromides with Butyl Vinyl Ether. Russ. J. Org. Chem. 2011, 47, 1594-1595. [CrossRef]

46. Musalov, M.V.; Potapov, V.A.; Kurkutov, E.O.; Musalova, M.V.; Khabibulina, A.G.; Amosova, S.V. Regioselective syntheses of bis-(2-haloalkyl) selenides and dihalo[bis-(2-haloalkyl)]- $\lambda 4$-selanes from selenium dihalides and 1-alkenes, and the methoxyselenenylation reaction. Arkivoc 2017, 2017, 365-376. [CrossRef]

47. Musalov, M.V.; Potapov, V.A.; Musalova, M.V.; Amosova, S.V. Stereoselective synthesis of (E,E)-bis(2-halovinyl) selenides and its derivatives based on selenium halides and acetylene. Tetrahedron 2012, 68, 10567-10572. [CrossRef]

48. Amosova, S.V.; Musalov, M.V.; Martynov, A.V.; Potapov, V.A. Regio- and Stereoselective Addition of Selenium Dihalogenides to Propargyl Halogenides. Russ. J. Gen. Chem. 2011, 81, 1239-1240. [CrossRef]

49. Musalov, M.V.; Martynov, A.V.; Amosova, S.V.; Potapov, V.A. Stereo- and Regioselective Reaction of Selenium Dichloride and Dibromide with Ethynyl(trimethyl)silane. Russ. J. Org. Chem. 2012, 48, 1571-1573. [CrossRef]

50. Potapov, V.A.; Musalov, M.V.; Musalova, M.V.; Rusakov, Y.Y.; Khabibulina, A.G.; Rusakova, I.L.; Amosova, S.V. Stereoselective synthesis of E-2-halovinyl tellanes, ditellanes and selenides based on tellurium tetrahalides, selenium dihalides and internal alkynes. J. Organomet. Chem. 2018, 867, 300-305. [CrossRef]

51. Musalov, M.V.; Potapov, V.A.; Amosova, S.V. Reaction of selenium dichloride with trimethylpropargylsilane. Russ. Chem. Bull. Int. Ed. 2011, 60, 769-770. [CrossRef]

52. Musalov, M.V.; Potapov, V.A.; Amosova, S.V. Reaction of Diselenium Dichloride with Acetylene. Russ. J. Org. Chem. 2011, 47, 1115-1116. [CrossRef]

53. Amosova, S.V.; Penzik, M.V.; Albanov, A.I.; Potapov, V.A. Addition of selenium dibromide to divinyl sulfide: Spontaneous rearrangement of 2,6-dibromo-1,4-thiaselenane to 5-bromo-2-bromomethyl-1,3-thiaselenolane. Tetrahedron Lett. 2009, 50, 306-308. [CrossRef]

54. Amosova, S.V.; Penzik, M.V.; Albanov, A.I.; Potapov, V.A. Synthesis of 2,6-Dichloro-1,4-thiaselenane from Divinyl Sulfide and Selenium Dichloride. Russ. J. Org. Chem. 2009, 45, 1271-1272. [CrossRef]

55. Potapov, V.A.; Amosova, S.V.; Volkova, K.A.; Penzik, M.V.; Albanov, A.I. Reactions of selenium dichloride and dibromide with divinyl selenide: Synthesis of novel selenium heterocycles and rearrangement of 2,6-dihalo-1,4-diselenanes. Tetrahedron Lett. 2010, 51, 89-92. [CrossRef]

56. Potapov, V.A.; Shagun, V.A.; Penzik, M.V.; Amosova, S.V. Quantum chemical studies of the reaction of selenium dichloride with divinyl sulfide and comparison with experimental results. J. Organomet. Chem. 2010, 695, 1603-1609. [CrossRef]

57. Potapov, V.A.; Volkova, K.A.; Penzik, M.V.; Albanov, A.I.; Amosova, S.V. Expedient Procedure for Preparation of 2-Chloromethyl1,3-diselenol. Russ. J. Gen. Chem. 2009, 79, 1225-1226. [CrossRef]

58. Potapov, V.A.; Volkova, K.A.; Penzik, M.V.; Albanov, A.I.; Amosova, S.V. Reaction of Selenium Dichloride with Divinyl Selenide. Russ. J. Org. Chem. 2008, 44, 1556-1557. [CrossRef]

59. Potapov, V.A.; Volkova, K.A.; Penzik, M.V.; Albanov, A.I.; Amosova, S.V. Synthesis of 4-Bromo-2-bromomethyl-1,3-diselenolane from Selenium Dibromide and Divinyl Selenide. Russ. J. Gen. Chem. 2008, 78, 1990-1991. [CrossRef]

60. Potapov, V.A.; Kurkutov, E.O.; Albanov, A.I.; Amosova, S.V. Regio- and Stereoselective Addition of Selenium Dibromide to Divinyl Sulfone. Russ. J. Org. Chem. 2008, 44, 1547-1548. [CrossRef]

61. Potapov, V.A.; Musalov, M.V.; Amosova, S.V.; Musalova, M.V.; Penzik, M.V. Reaction of Selenium Dichloride with Divinyl Telluride. Russ. J. Org. Chem. 2011, 47, 950-951. [CrossRef] 
62. Potapov, V.A.; Kurkutov, E.O.; Amosova, S.V. Synthesis of a New Four-Membered Heterocycle by Reaction of Selenium Dichloride with Divinyl Sulfone. Russ. J. Org. Chem. 2010, 46, 1099-1100. [CrossRef]

63. Trofimov, B.A.; Amosova, S.V. Divinil Sulfid i Ego Proizvodnye (Divinyl Sulfide and Its Derivatives); Nauka: Novisibirsk, Russia, 1983; p. 264. (In Russian)

64. Gusarova, N.K.; Trofimov, B.A.; Potapov, V.A.; Amosova, S.V.; Sinegovskaya, L.M. Reactions of Elemental Selenium with Acetylenes. 1. Identification of Products of Reaction of Elemental Selenium with Acetylene. Zhurnal Org. Khimii 1984, 20, 484-489. (In Russian)

65. Potapov, V.A.; Gusarova, N.K.; Amosova, S.V.; Kashik, A.S.; Trofimov, B.A. Reactions of Chalcogen with Acetylenes. 2. Reaction of Selenium Metals with Acetylene in the HMPA and DMSO Media. Zhurnal Org. Khimii 1986, 22, 276-281. (In Russian)

66. Musalov, M.V.; Potapov, V.A.; Amosova, S.V.; Musalova, M.V.; Volkova, K.A. Reactions of Selenium Dichloride and Dibromide with Diallyl Telluride. Russ. J. Gen. Chem. 2011, 81, 2201-2202. [CrossRef]

67. Potapov, V.A.; Amosova, S.V.; Abramova, E.V.; Musalov, M.V.; Lyssenko, K.A.; Finn, M.G. 2,6-Dihalo-9-selenabicyclo[3.3.1]nonanes and their complexes with selenium dihalides: Synthesis and structural characterization. New J. Chem. 2015, 39, 8055-8059. [CrossRef]

68. Abramova, E.V.; Sterkhova, I.V.; Molokeev, M.S.; Potapov, V.A.; Amosova, S.V. First coordination compounds of SeBr 2 with selenium ligands: X-ray structural determination. Mendeleev Commun. 2016, 26, 532-534. [CrossRef]

69. Yurieva, O.V.; Dubrovina, V.I.; Potapov, V.A.; Musalov, M.V.; Starovoitova, T.P.; Ivanova, T.A.; Gromova, A.V.; Shkaruba, T.T.; Balakhonov, S.V. Effect of Synthetic Organoselenium Drug on the Degree of Pathological Changes in the Organs of White Mice Immunized with Tularemia and Brucellosis Vaccines. Bull. Exp. Biol. Med. 2019, 168, 66-69. [CrossRef]

70. Nesterkina, I.S.; Musalov, M.V.; Gurina, V.V.; Ozolina, N.V.; Spiridonova, E.V.; Tretyakova, A.V.; Potapov, V.A.; Amosova, S.V.; Yakimov, V.A. The effect of a new non-toxic water-soluble selenorganic substance on antioxidant protection and development of seedlings of oilseed radish (Raphanus sativus L. var. oleiferus Metzg.). Acta Agric. Slov. 2019, 114, 61-67. [CrossRef]

71. Wang, J.; Sanchez-Rosello, M.; Aceña, J.L.; del Pozo, C.; Sorochinsky, A.E.; Fustero, S.; Soloshonok, V.A.; Liu, H. Fluorine in Pharmaceutical Industry: Fluorine-Containing Drugs Introduced to the Market in the Last Decade (2001-2011). Chem. Rev. 2014, 114, 2432-2506. [CrossRef] [PubMed] 